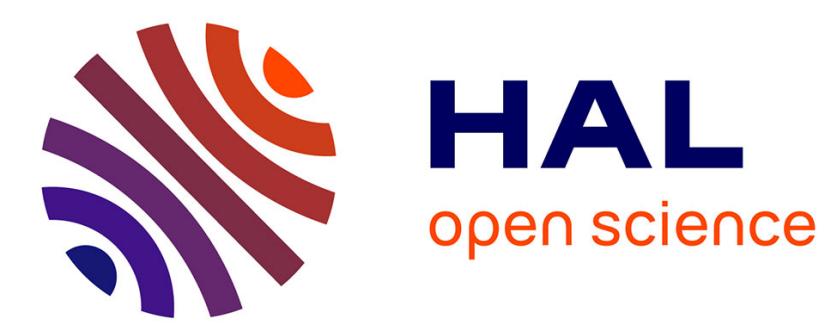

\title{
About geometrically maximal manifolds
}

Charles Frances

\section{To cite this version:}

Charles Frances. About geometrically maximal manifolds. Journal of topology, 2012, 5 (2), pp.293322. 10.1112/JTOPOL/JTS003 . hal-03165730

\section{HAL Id: hal-03165730 \\ https://hal.science/hal-03165730}

Submitted on 10 Mar 2021

HAL is a multi-disciplinary open access archive for the deposit and dissemination of scientific research documents, whether they are published or not. The documents may come from teaching and research institutions in France or abroad, or from public or private research centers.
L'archive ouverte pluridisciplinaire HAL, est destinée au dépôt et à la diffusion de documents scientifiques de niveau recherche, publiés ou non, émanant des établissements d'enseignement et de recherche français ou étrangers, des laboratoires publics ou privés. 


\title{
About geometrically maximal manifolds
}

\author{
Charles Frances
}

\section{Introduction}

Given a geometric structure $\mathcal{S}_{M}$ on a noncompact manifold $M$, it is a natural question to know wether the structure $\mathcal{S}_{M}$ can be "prolongated at infinity". In other words, we look for a manifold $N$ having the same dimension as $M$, a geometric structure $\mathcal{S}_{N}$ on $N$ of the same kind of $\mathcal{S}_{M}$, and a strict geometric embedding $\phi:\left(M, \mathcal{S}_{M}\right) \rightarrow\left(N, \mathcal{S}_{N}\right)$. By a geometric embedding, we mean that $\phi^{*} \mathcal{S}_{N}=\mathcal{S}_{M}$, namely the pullback of the structure $\mathcal{S}_{N}$ is $\mathcal{S}_{M}$. The embedding is called strict when it is not onto.

The question comprises two aspects. The first one is to find criteria on a given structure $\left(M, S_{M}\right)$ ensuring that a strict geometric embedding exists. The second one is, on the contrary, to look for structures $\left(M, S_{M}\right)$ which are geometrically maximal, in the sense that every geometric embedding $\phi:\left(M, S_{M}\right) \rightarrow\left(N, S_{N}\right)$ must be onto.

Regarding the first aspect, very interesting works were done in the realm of Riemannian conformal structures. In this context, our question becomes simply: "when is a Riemannian manifold $(M, g)$ conformally equivalent to some proper open subset of another Riemannian manifold $(N, h)$ ?" For example, in $[\mathrm{S}-\mathrm{Y}]$, the authors exhibit conditions on a $n$-dimensional conformally flat Riemannian manifold $(M, g)$, ensuring that $(M, g)$ can be embedded conformally as an open subset of the standard sphere $\mathbf{S}^{n}$ (see in particular [S-Y], theorem 3.1). In the same spirit, it is shown in $[\mathrm{C}-\mathrm{H}]$ that under suitable conditions involving the Ricci and the scalar curvature, a complete conformally flat manifold $(M, g)$ is conformally equivalent to a compact orbifold with a finite number of singular points removed. Among other relevant results about conformal embeddings and compactification of Riemannian manifolds, let us also quote [Cha] and [He1].

In this paper, we will be interested by the rather opposite aspect, namely find quite general conditions ensuring that a geometric structure $\left(M, \mathcal{S}_{M}\right)$ is geometrically maximal. Of course, we will implicitly deal with noncompact manifolds, since any structure on a compact manifold is maximal for obvious topological reasons. Geometric maximality can be seen as a weak notion of completeness for our geometric structure $\left(M, S_{M}\right)$.

The main results we obtain are theorems 1.1, 1.4, 6.4, 7.3 and 9.1. The first one exhibits examples of conformally maximal manifolds: essentially 
all Riemannian homogeneous spaces of dimension $n \geq 3$ are conformally maximal, the only exceptions being the obvious ones, namely open subsets of the sphere which are homogeneous under the action of a subgroup of the Möbius group. An analogue result holds for strictly pseudoconvex $C R$ structures (theorem 9.1). Theorem 7.3 gives obstructions, for a class of geometric structures including Riemannian conformal, and $C R$-structures, to admit a geometric compactification.

Part of the techniques used in the paper can be applied to quite a wide class of geometric structures called Cartan geometries. A lot of classical geometries, such as pseudo-Riemannian metrics, pseudo-Riemannian conformal structures, $C R$-structures, affine or projective structures are Cartan geometries. Our theorem 1.4 yields a maximality criteria for an interesting class of Cartan geometries: the so called Kleinian manifolds. Concrete applications of the theorem will be listed in corollaries 1.5, 1.6 and section 5.2 .

We now describe our results in more details, emphasizing the most familiar context of Riemannian conformal structures.

\subsection{Conformally maximal manifolds}

In the metric context, it is quite clear that a geodesically complete pseudoRiemannian manifold $(M, g)$ is maximal in the sense that any isometric embedding $s:(M, g) \rightarrow(N, h)$, where $(N, h)$ is pseudo-Riemannian of the same type as $(M, g)$, has to be onto. In particular, any $n$-dimensional Riemannian homogeneous space $(M, g)$ is maximal among $n$-dimensional Riemannian manifolds.

On the contrary, completeness of a Riemannian manifold $(M, g)$ is no longer a sufficient condition to ensure conformal maximality. We already see this with the $n$-Euclidean space, which admits a strict conformal embedding into the standard sphere $\mathbf{S}^{n}$ thanks to the stereographic projection. Actually, the notion of conformal maximality is rather subtle, and to our knowledge, we don't have a wide list of Riemannian or pseudo-Riemannian structures which are notoriously conformally maximal. This is a motivation for the following:

Theorem 1.1. Let $(M, g)$ be a Riemannian manifold of dimension $n \geq 3$, which is conformally homogeneous. Then it is conformally maximal except in the following cases:

1. $(M, g)$ is conformally equivalent to the Euclidean space $\mathbf{R}^{n}$.

2. $(M, g)$ is conformally equivalent to the real hyperbolic space $\mathbf{H}^{n}$. 
3. $(M, g)$ is conformally equivalent to the product $\mathbf{H}^{m} \times \mathbf{S}^{k}$, with $m \geq 1$, $k \geq 1, m+k \geq 3$ (with the convention that $\mathbf{H}^{1}$ is the 1-dimensional Euclidean space and $\mathbf{S}^{1}$ is the circle endowed with the homogeneous Riemannian metric of total length $2 \pi$ ).

The terminology conformally homogeneous means that the conformal group of $(M, g)$, namely the group of smooth diffeomorphisms $\phi: M \rightarrow M$ such that $\phi^{*} g=e^{\alpha} g$ with $\alpha$ a smooth function, acts transitively on $M$. Let us also emphasize that in the statement above, the products $\mathbf{H}^{m} \times \mathbf{S}^{k}, m \geq 2$, $k \geq 2$, are endowed with the conformal class of the "natural" product metric, namely we put the hyperbolic metric, with constant curvature -1 , on the factors $\mathbf{H}^{m}$ and the complete metric of curvature +1 on the factors $\mathbf{S}^{k}$. Stated differently, theorem 1.1 says that conformally homogeneous Riemannian manifolds of dimension $\geq 3$ which are not conformally maximal are conformally equivalent to open subsets of $\mathbf{S}^{n}$, obtained by removing one point, two points, or a codimension $k$ conformal sphere, $1 \leq k \leq n-1$.

From theorem 1.1 we infer, for example, that given a Lie group $G$ which does not act simply transitively on one of the spaces listed in the theorem, and given a left invariant Riemannian metric $g$ on $G$, then $(G, g)$ is a conformally maximal Riemannian manifolds. This is for instance the case if $G$ is a noncompact simple Lie group. It is worth noticing that this fact is no longer true if we consider pseudo-Riemannian metrics. For instance, on the group $G=P S L(2, \mathbf{R})$, the Killing form has Lorentz signature, hence defines a bi-invariant Lorentz metric on $P S L(2, \mathbf{R})$. This Lorentz manifold, known as the 3-dimensional anti-de Sitter space, is not conformally maximal. It can be embedded conformally in the product $\mathbf{S}^{1} \times \mathbf{S}^{2}$, endowed with the conformal class of the metric $-d t^{2}+g_{S^{2}}$.

Another consequence of theorem 1.1 is that Riemannian symmetric spaces of noncompact type, except the real hyperbolic space $\mathbf{H}^{n}$, are conformally maximal in dimension $\geq 3$. For example, it is not possible to embed conformally the complex hyperbolic space as a proper open subset of a Riemannian manifold.

\section{Obstruction to conformal compactifications}

Among conformal embeddings, the most interesting ones are maybe those for which the target manifold is compact. If $(M, g)$ is a Riemannian manifold, and if there exists a closed Riemannian manifold $(N, h)$, as well as a conformal embedding $s:(M, g) \rightarrow(N, h)$, then we say that $(N, h)$ is a conformal compactification of $(M, g)$. For example, the standard sphere $\mathbf{S}^{n}$ is a conformal compactification for the $n$-dimensional Euclidean and (real) 
hyperbolic spaces. Observe that this definition is more general than the classical notion of conformally compact manifold $(M, g)$, where $(M, g)$ is required to be the interior of a manifold with boundary $\bar{M}$, the conformal structure of $g$ extending smoothly to the boundary. In all the paper, and especially in the definition of conformal compactification given above, the target manifold $N$ is without boundary, and we don't make any assumption on the topological boundary of $s(M) \subset N$. This boundary is allowed to be very irregular.

In [Ki], S. Kim showed that if a certain numerical conformal invariant of a manifold $(M, g)$ is small enough, then $(M, g)$ does not admit any conformal compactification. Here, we give an obstruction to the conformal compactification of a noncompact Riemannian manifold $(M, g)$, involving its isometry group.

Theorem 1.2. Let $(M, g)$ be a connected Riemannian manifold of dimension $n \geq 3$. Assume that the isometry group of $(M, g)$ is noncompact, and that $(M, g)$ is not conformally flat. Then, there is no conformal compactification for $(M, g)$.

As an example, we see that for "generic" Riemannian manifolds $M$, a Riemannian product $\mathbf{R} \times M$ does not admit any conformal compactification. Theorem 1.2 is actually a particular case of the more general theorem 7.3.

\subsection{Maximal Cartan geometries}

Until now, we decribed examples of conformally maximal manifolds, but as we already mentioned, the issue of maximality makes sense for general geometric structures. In this paper, we chose to tackle this problem in the framework of Cartan geometries. This is quite a wide family of geometries, comprising most of those which are usually called rigid geometric structures. For the reader which is not familiar with this notion, let us emphasize that pseudo-Riemannian metrics, pseudo-Riemannian conformal structures, nondegenerate $C R$-structures, affine structures, projective structures are Cartan geometries. Thus, beyond the rather abstract setting, we will always keep in mind those basic and classical examples. Above all, the language of Cartan geometries will be absolutely fundamental in all the proofs of our main results, including theorems 1.1 and 9.1 about Riemannian conformal, and $C R$-structures. 


\subsubsection{Cartan geometries}

We refer to [Sh] for a comprehensive introduction to the notion of Cartan geometry. Very roughly, a Cartan geometry is a geometric structure whose flat model is a homogeneous space. The precise definition is as follows.

Let $G$ be a Lie group, $P \subset G$ a closed subgroup, and $\mathbf{X}=G / P$. In all the paper, we will do the assumption that $\mathbf{X}$ is connected and $G$ acts faithfully on $\mathbf{X}$. A homogeneous space $\mathbf{X}=G / P$ satisfying those conditions will be referred to as a model space. One calls a Cartan geometry modelled on $\mathbf{X}$ the data of a triple $(M, \hat{M}, \omega)$, where:

1. $M$ is a connected manifold having the same dimension as $\mathbf{X}$.

2. $\hat{M}$ is a $P$-principal bundle over $M$.

3. The form $\omega$ is a 1-form taking values in $\mathfrak{g}$, and satisfying:

- For every $\hat{x} \in \hat{M}, \omega_{\hat{x}}: T_{\hat{x}} \hat{M} \rightarrow \mathfrak{g}$ is an isomorphism of vector spaces.

- For every $X \in \mathfrak{p}$ and $\hat{x} \in \hat{M}, \omega_{\hat{x}}\left(\frac{d}{d t \mid t=0} R_{e^{t X}} . \hat{x}\right)=X$.

- For every $p \in P,\left(R_{p}\right)^{*} \omega=\left(\operatorname{Ad} p^{-1}\right) . \omega$.

Here, $R_{p}$ denotes the right action by $p$ on $\hat{M}$, and $\left\{e^{t X}\right\}$ is the 1-parameter group of $G$ determined by $X$. A 1 -form $\omega$ as above is called a Cartan connexion on $\hat{M}$.

The flat model is the triple $\left(\mathbf{X}, G, \omega^{G}\right)$, where $\omega^{G}$ is the Maurer-Cartan form on $G$. Actually, there is, on the fiber bundle $\hat{M}$, a notion of curvature, which is a $P$-equivariant map $\kappa: \hat{M} \rightarrow \operatorname{Hom}\left(\Lambda^{2}(\mathfrak{g} / \mathfrak{p})^{*}, \mathfrak{g}\right)$ (see [Sh, p. 191]). This curvature map $\kappa$ vanishes on $\hat{M}$ if and only if the bundle $\hat{M} \rightarrow M$ is locally isomorphic to $G \rightarrow \mathbf{X}$, and the local isomorphisms $\varphi$ satisfy $\varphi^{*}\left(\omega^{G}\right)=\omega$ (see $[$ Sh, 5.5]). One then says that $(M, \hat{M}, \omega)$ is flat. In other words, among Cartan geometries $(M, \hat{M}, \omega)$ modelled on $\mathbf{X}$, the flat ones correspond to $(G, \mathbf{X})$-structure on $M$ (see [Th, 3.3] for an introduction to this notion). All the Cartan geometries considered in this paper will always supposed to be smooth.

\subsubsection{Geometric immersions, geometric embeddings}

Let $\mathbf{X}=G / P$ be a model space, and let us consider $\left(M, \hat{M}, \omega^{M}\right)$ and $\left(N, \hat{N}, \omega^{N}\right)$ two Cartan geometries modelled on $\mathbf{X}$ (notice that $M$ and $N$ automatically have the same dimension, which is that of $\mathbf{X}$ ). 
By a geometric immersion $\sigma$ of $\left(M, \hat{M}, \omega^{M}\right)$ into $\left(N, \hat{N}, \omega^{N}\right)$, we mean a smooth bundle map $\sigma: \hat{M} \rightarrow \hat{N}$, which is $P$-equivariant and such that $\sigma^{*}\left(\omega^{N}\right)=\omega^{M}$. (Observe that the condition $\sigma^{*}\left(\omega^{N}\right)=\omega^{M}$ implies that the differential of $\sigma$ is an isomorphism at each point of $\hat{M})$. Such an immersion induces a smooth immersion $s: M \rightarrow N$. We will say that $\sigma$ is a strict immersion if $\sigma(\hat{M})$ is a proper open subset of $\hat{N}$ (or equivalently $s(M)$ is a proper open subset of $N$ ). If the map $\sigma$ is moreover injective, we will speak of a geometric embedding, and when a geometric embedding $\sigma$ is not strict, we just have a geometric isomorphism between $\left(M, \hat{M}, \omega^{M}\right)$ and $\left(N, \hat{N}, \omega^{N}\right)$. In this case $\left(M, \hat{M}, \omega^{M}\right)$ and $\left(N, \hat{N}, \omega^{N}\right)$ are the same from the point of view of Cartan geometries modelled on $\mathbf{X}$. The group of geometric isomorphisms from $\left(M, \hat{M}, \omega^{M}\right)$ to $\left(M, \hat{M}, \omega^{M}\right)$ is called the automorphism group of $\left(M, \hat{M}, \omega^{M}\right)$, and denoted by $\operatorname{Aut}\left(\hat{M}, \omega^{M}\right)$. By definition, an automorphism of $\left(M, \hat{M}, \omega^{M}\right)$ commutes with the action of $P$, so that $\operatorname{Aut}\left(\hat{M}, \omega^{M}\right)$ induces on $M$ a subgroup of $\operatorname{Diff}(M)$, denoted by $\operatorname{Aut}\left(M, \omega^{M}\right)$. Our assumption that the action of $G$ is faithfull on $\mathbf{X}$ implies that any element of $\operatorname{Aut}\left(M, \omega^{M}\right)$ lifts uniquely to an element of $\operatorname{Aut}\left(\hat{M}, \omega^{M}\right)$.

The definition of geometric embeddings endows naturally the set of Cartan geometries modelled on a given $\mathbf{X}=G / P$ with a partial ordering. If $\left(M, \hat{M}, \omega^{M}\right)$ and $\left(N, \hat{N}, \omega^{N}\right)$ are two such geometries, we will say that $M$ is smaller than $N$ if there is a strict geometric embedding $\sigma:\left(M, \hat{M}, \omega^{M}\right) \rightarrow$ $\left(N, \hat{N}, \omega^{N}\right)$. This leads to the following definition.

Definition 1.3. (Maximal and strongly maximal Cartan geometries)

A Cartan geometry $\left(M, \hat{M}, \omega^{M}\right)$ modelled on $\mathbf{X}$ is geometrically maximal (resp. strongly geometrically maximal) when every geometric embedding (resp. immersion) from $\left(M, \hat{M}, \omega^{M}\right)$ into any Cartan geometry $\left(N, \hat{N}, \omega^{N}\right)$ modelled on $\mathbf{X}$ is onto.

\subsubsection{A maximality result for Kleinian manifolds}

Let $\mathbf{X}=G / P$ be a model space. Assume that $\Omega$ is an open subset of $\mathbf{X}$, and $\Gamma \subset G$ is a discrete subgroup acting freely and properly on $\Omega$. Then the manifold $M_{\Gamma}:=\Gamma \backslash \Omega$ is called a Kleinian manifold. As we will see in section 3.1, a Kleinian manifold is naturally endowed with a flat Cartan geometry modelled on $\mathbf{X}$.

As an example, if $\mathbf{X}=P O(1, n+1) / P$ is the $n$-dimensional standard conformal sphere, then complete hyperbolic manifolds are interesting examples of Kleinian manifolds. In this case, $\Omega$ is the upper hemisphere, and the natural flat Cartan geometry is the conformally flat structure defined by the hyperbolic metric. 
The following theorem gives a sufficient condition, ensuring that a Kleinian manifold is strongly geometrically maximal, among all Cartan geometries modelled on $\mathbf{X}$.

Theorem 1.4. Let $\Omega \subsetneq \mathbf{X}$ be a normal domain. Let $M_{\Gamma}=\Gamma \backslash \Omega$ be a Kleinian manifold. If the action of $\Gamma$ is free and proper on no open subset of $\bar{\Omega}$ properly containing $\Omega$, then $M_{\Gamma}$ is strongly geometrically maximal among all the Cartan geometries modelled on $\mathbf{X}$.

The notion of normal domain will be introduced in section 3.2. Intuitively, a normal domain is an open set whose boundary is not too wild. For example, open subsets whose boundary is a locally Lipschitz hypersurface, or have Hausdorff codimension $>1$ are normal.

It is interesting to apply theorem 1.4 for various models $\mathbf{X}$, and various groups $\Gamma$, getting a lot of examples of maximal structures, in different geometric situations. The reader who is eager to see concrete applications is referred to section 5.2. For the moment, let us just see two direct consequences for conformal Riemannian structures (the proofs will be given in $5.2)$.

Corollary 1.5. Every flat complete Riemannian manifold of dimension $n \geq$ 3, except the Euclidean space, is strongly conformally maximal.

This corollary does not extend to general pseudo-Riemannian structures. In Lorentz signature, Minkowski's space can be embedded conformally in a compact Lorentz manifold called Einstein's universe. But it is not the only flat complete Lorentz manifold to have this property. For instance, one can show that the quotient of Minkowski's space by the subgroup generated by one translation is never conformally maximal. There are also much more interesting complete flat Lorentz manifolds, known as Margulis space-times (see $[\mathrm{D}],[\mathrm{Ma}]$ ), which admit strict conformal embeddings (see [Fr3]).

Corollary 1.6. For a complete hyperbolic manifold $M=\Gamma \backslash \mathbf{H}^{n}, n \geq 3$, the following conditions are equivalent:

1. $M$ is strongly conformally maximal.

2. The limit set $\Lambda_{\Gamma}$ is equal to $\mathbf{S}^{n-1}=\partial \mathbf{H}^{n}$.

We refer to [Ka, Chap. 4] for the definition of the limit set of a subgroup of Is $\left(\mathbf{H}^{n}\right)$. The corollary implies in particular that complete hyperbolic manifolds of finite volume and dimension $\geq 3$ are strongly conformally maximal. 
This fact is not true in dimension 2: some hyperbolic surfaces of finite volume are conformally equivalent to compact Riemann surfaces with a finite number of points removed.

Let us emphasize that corollaries 1.5 and 1.6 (at least in the finite volume case) are certainly already known. We refer especially to [C-H, Theorems 2.1, 5.7 and 5.9], for an analytical approach.

\subsection{Organization of the paper}

The article will begin with a general construction of a boundary for any Cartan geometry. Although this boundary is generally hard to understand, we can describe it explicitly in the case of Kleinian manifolds, what will be done in section 3 . Then, in section 4, we will explain how any geometric immersion extends to part of the Cauchy boundary, thus defining a boundary map. The properties of this map will be derived in proposition 4.4. Thanks to this preliminary work, we will be able to prove theorem 1.4 in section 5 . We will give several illustrations of this theorem in 5.2. Other maximality criteria are proved in section 6, especially theorem 6.4 and corollary 6.6. With section 7 begins the second part of the paper, and the description of homogeneous conformal structures (resp. $C R$-structures) which are not maximal. A first step is proposition 7.1, which shows that such structures are actually flat. The last two sections are then devoted to the proofs of theorems 1.1 and 9.1, using the classification of homogeneous conformally flat structures by Alekseevskii and Kimel'fel'd on the one hand, and the classification of homogeneous domains of the flat $C R$-sphere by Burns and Schnider on the other hand. Notice that those proofs heavily rely on the results obtained in sections 5 and 6 .

\section{The Cauchy boundary of a Cartan geometry}

Our aim in this section is to attach an abstract boundary to any Cartan geometry. The construction is a straightforward generalization of the so called b-boundary, first introduced by B.G. Schmidt in [S].

\subsection{Cauchy completion of a Cartan geometry}

We consider a model space $\mathbf{X}=G / P$, and a Cartan geometry $\left(M, \hat{M}, \omega^{M}\right)$ modelled on $\mathbf{X}$. Let us fix once for all $X_{1}, \ldots, X_{m}$ a basis of the Lie algebra $\mathfrak{g}$. This choice defines a frame field $\mathcal{R}$ on $\hat{M}$ as follows

$$
\mathcal{R}_{\hat{x}}=\left(\left(\omega_{\hat{x}}^{M}\right)^{-1}\left(X_{1}\right), \ldots,\left(\omega_{\hat{x}}^{M}\right)^{-1}\left(X_{m}\right)\right), \quad \hat{x} \in \hat{M}
$$


There is a unique Riemannian metric $\rho^{M}$ on $\hat{M}$ for which the frame field $\mathcal{R}$ is orthonormal. If $\hat{x}$ and $\hat{y}$ are in a same connected component of $\hat{M}$, and if $\Gamma(\hat{x}, \hat{y})$ is the set of $C^{1}$ maps $\gamma:[0,1] \rightarrow \hat{M}$ such that $\gamma(0)=\hat{x}$ and $\gamma(1)=\hat{y}$, then define

$$
\delta_{M}(\hat{x}, \hat{y}):=\inf \left\{\int_{0}^{1} \sqrt{\rho^{M}\left(\gamma^{\prime}(t), \gamma^{\prime}(t)\right)} d t \mid \gamma \in \Gamma(\hat{x}, \hat{y})\right\}
$$

We define a distance $d_{M}$ on $\hat{M}$ by the formula $d_{M}(\hat{x}, \hat{y})=\frac{\delta_{M}(\hat{x}, \hat{y})}{1+\delta_{M}(\hat{x}, \hat{y})}$ if $\hat{x}$ and $\hat{y}$ are in a same component of $\hat{M}$, and $d_{M}(\hat{x}, \hat{y})=2$ if $\hat{x}$ and $\hat{y}$ are in different components. Let $\left(\hat{M}_{c}, \bar{d}_{M}\right)$ denote the Cauchy completion of the metric space $\left(\hat{M}, d_{M}\right)$, and $\partial_{c} \hat{M}$ its Cauchy boundary, i.e $\partial_{c} \hat{M}=\hat{M}_{c} \backslash \hat{M}$. Let us remark that for every $p \in P$, the Jacobian matrix of $R_{p}$, the right multiplication by $p$, when expressed in the frame field $\mathcal{R}$, is exactly the matrix of the linear map Ad $p^{-1}$ expressed in the basis $X_{1}, \ldots, X_{m}$. It follows that the maps $R_{p}$ are Lipschitz with respect to $d_{M}$, thus extend to continuous maps $\bar{R}_{p}$ on $\hat{M}_{c}$. Those maps $\bar{R}_{p}$ leave $\partial_{c} \hat{M}$ invariant. Observe that for every compact set $K \subset P$, the norms of the transformations Ad $p^{-1}$ are uniformly bounded when $p \in K$. As a consequence, there exists $L_{K}>0$ such that for every $p \in K$, the transformation $\bar{R}_{p}$ is $L_{K}$-Lipschitz. It follows that the action $P \times \hat{M}_{c} \rightarrow \hat{M}_{c}$ is continuous.

One can also define the Cauchy completion $M_{c}$ (resp. the Cauchy boundary $\partial_{c} M$ ) of $M$ as the quotient $\hat{M}_{c} / P$ (resp. $\partial_{c} \hat{M} / P$ ). The space $M_{c}$ may not be a nice topological space, because the action of $P$, which is proper on $\hat{M}$, can behave very badly on the boundary $\partial_{c} \hat{M}$, yielding a non Hausdorff quotient.

In the definition of the Cauchy boundary, the initial choice of the basis $X_{1}, \ldots, X_{m}$ is not relevant, since different choices lead to bi-Lipschitz equivalent metrics, hence to the same Cauchy completion.

\subsection{Some remarks about completeness}

As a very simple first illustration of the previous construction, let us consider a model space $\mathbf{X}=G / P$, and the triple $\left(\mathbf{X}, G, \omega^{G}\right)$, where $\omega^{G}$ denotes the Maurer-Cartan form on $G$. This is the canonical flat Cartan geometry modelled on $\mathbf{X}$. If $X_{1}, \ldots, X_{m}$ is a basis of $\mathfrak{g}$, and $\rho^{G}$ is the Riemannian metric on $G$ making the frame field $\left(\omega^{G}\right)^{-1}\left(X_{1}, \ldots, X_{m}\right)$ orthonormal, then $\rho^{G}$ is left-invariant because $\omega^{G}$ itself is left-invariant. Hence, $\left(G, \rho^{G}\right)$ is a homogeneous Riemannian manifold and, in particular, each connected component of $G$ is complete for the distance $\delta_{G}$ defined in the previous 
section. The metric space $\left(G, d_{G}\right)$ is itself complete and the Cauchy boundary of this canonical structure is empty.

It is worth noticing that we don't know any characterization of complete Cartan geometries, i.e those $\left(M, \hat{M}, \omega^{M}\right)$ for which $\partial_{c} M=\emptyset$, except in the realm of Riemannian metrics. In particular, the compactness of $M$ is generally not sufficient to ensure completeness (see for instance remark 3.6, where we see that the conformal Cauchy boundary of a flat Riemannian torus is a point). The relationship between different notions of completeness is clarified in [Cl]. See also [MK1] for a description of complete complex parabolic geometries. In his work, though, McKay is considering a stronger notion of completeness.

Let us discuss now an a priori weaker notion of completeness, for a Cartan geometry $\left(M, \hat{M}, \omega^{M}\right)$. The data of $X$ in $\mathfrak{g}$ defines naturally a $\omega^{M}$-constant vector field $\hat{X}$ on $\hat{M}$ by the relation $\omega^{M}(\hat{X})=X$. We denote by $\phi_{X}^{t}$ the local flow generated on $\hat{M}$ by the field $\hat{X}$. For each $\hat{x} \in \hat{M}$, we define $\mathcal{W}_{\hat{x}} \subset \mathfrak{g}$ as the set of vectors $X \in \mathfrak{g}$ such that $\phi_{X}^{t} . \hat{x}$ is defined for $t \in[0,1]$. Then one defines the exponential map at $\hat{x}$ as

$$
\exp (\hat{x}, X):=\phi_{X}^{1} \cdot \hat{x}, \forall X \in \mathcal{W}_{\hat{x}}
$$

Let us mention the nice behavior of this map with respect to geometric immersions. If $\left(M, \hat{M}, \omega^{M}\right)$ and $\left(N, \hat{N}, \omega^{N}\right)$ are two Cartan geometries modelled on $\mathbf{X}$, and if $\sigma: \hat{M} \rightarrow \hat{N}$ is a geometric immersion, then $\sigma$ maps $\omega$-constant vector fields of $\hat{M}$ to $\omega$-constant vector fields of $\hat{N}$. As a consequence, for every $\hat{x} \in \hat{M}$, we have $\mathcal{W}_{\hat{x}} \subset \mathcal{W}_{\sigma(\hat{x})}$, and for every $X \in \mathcal{W}_{\hat{x}}$

$$
\sigma(\exp (\hat{x}, X))=\exp (\sigma(\hat{x}), X)
$$

Observe that the exponential map exp is generally different from the exponential defined by the Riemannian metric $\rho^{M}$.

The link between the completeness of the metric space $\left(\hat{M}, d_{M}\right)$, and the fact that $X \mapsto \exp (\hat{x}, X)$ is defined on $\mathfrak{g}$ for every $\hat{x} \in \hat{M}$, is an interesting question that is still far from being clarified. It motivates the following definition, which will be useful later on.

Definition 2.1 (Boundary points of the first kind). Let $\left(M, \hat{M}, \omega^{M}\right)$ be a Cartan geometry modelled on $\mathbf{X}=G / P$, and let $\partial_{c} \hat{M}$ be its Cauchy boundary. A point $\hat{z} \in \partial_{c} \hat{M}$ is said to be of the first kind if there exists $X \in \mathfrak{g}$, $\hat{x} \in \hat{M}$, such that the curve $t \mapsto \exp (\hat{x}, t X)$ is defined on $[0, a[, a>0$, and $\lim _{t \rightarrow a^{-}} \exp (\hat{x}, t X)=\hat{z}$. 


\section{First examples of Cauchy boundaries: the case of Kleinian manifolds}

Let us mention that we don't know very many Cartan geometries for which the Cauchy boundary is understood. (See [Cla], [Bo], [J] for works in Lorentz geometry). Nevertheless, there is a quite wide family of structures for which the determination of this boundary is possible, and we describe them below.

\subsection{Canonical Cartan geometry on a Kleinian manifold}

We consider a model space $\mathbf{X}=G / P$, and the triple $\left(\mathbf{X}, G, \omega^{G}\right)$, where $\omega^{G}$ denotes the Maurer-Cartan form on $G$. The choice of a basis $X_{1}, \ldots, X_{m}$ of $\mathfrak{g}$ determines a Riemannian metric $\rho^{G}$ on $G$ as explained in the previous section. We observed that $\rho^{G}$ is left-invariant, and $\left(G, d_{G}\right)$ is a complete metric space.

Let now $\Omega$ be an open subset of $\mathbf{X}$, and $\Gamma \subset G$ a discrete subgroup acting freely properly and discontinuously on $\Omega$. The Kleinian manifold $M=\Gamma \backslash \Omega$ is naturally endowed with a flat Cartan geometry, inherited from $\left(\mathbf{X}, G, \omega^{G}\right)$. Let us explain this in detail. We denote by $G_{\Gamma}$ the quotient $\Gamma \backslash G$. Since $\omega^{G}$ is left-invariant, it induces a 1 -form $\omega^{G_{\Gamma}}$ on $G_{\Gamma}$, with values in $\mathfrak{g}$. The manifold $G_{\Gamma}$ carries a right action of $P$, and the form $\omega^{G_{\Gamma}}$ fullfills all the conditions of a Cartan connection, listed in 1.2.1. Nevertheless, we don't have a Cartan geometry in the classical sense because $G_{\Gamma}$ is generally not a $P$-principal bundle. Indeed, as soon as $\Gamma$ does not act freely and properly on $\mathbf{X}$, the $P$-action on $G_{\Gamma}$ will neither be free nor proper. Such a pair $\left(G_{\Gamma}, \omega^{G_{\Gamma}}\right)$ will be referred to as a weak Cartan geometry.

Let $\pi_{X}: G \rightarrow \mathbf{X}$ be the bundle projection. If $\hat{\Omega}$ denotes the inverse image of $\Omega$ by $\pi_{X}$, the Maurer-Cartan form $\omega^{G}$ restricts to $\hat{\Omega}$ giving a 1 -form $\omega^{\Omega}$. The open subset $\hat{\Omega}$, as well as the form $\omega^{\Omega}$, are left invariant by the leftaction of $\Gamma$, so that the form $\omega^{\Omega}$ induces a 1 -form $\omega^{M}$ with values in $\mathfrak{g}$ on the manifold $\hat{M}:=\Gamma \backslash \hat{\Omega}$. This time, $\hat{M}$ is a $P$-fiber bundle over $M$, and the triple $\left(M, \hat{M}, \omega^{M}\right)$ is a Cartan geometry modelled on $\mathbf{X}$. It will be called the canonical Cartan geometry of the Kleinian manifold $M$.

Observe that $\hat{M}$ is naturally identified with an open subset of $G_{\Gamma}$, and that $\omega^{M}$ is just the restriction of $\omega^{G_{\Gamma}}$ to $\hat{M}$. Our aim in the following will be to understand the relationship between $\partial \hat{M}$, the topological boundary of $\hat{M}$ in $G_{\Gamma}$, and the Cauchy boundary $\partial_{c} \hat{M}$ of the Cartan geometry $\left(M, \hat{M}, \omega^{M}\right)$. This relationship will be very clear when the boundary of the open subset $\Omega$ does not behave too wildly. This leads to the notion of normal domain that we study below. 


\subsection{Normal domains}

Let $Y$ be a smooth manifold and $W \subsetneq Y$ an open subset. We denote by $\bar{W}$ the closure of $W$ in $Y$, and define the topological boundary of $W$, denoted $\partial W$, as the complement of $W$ inside $\bar{W}$.

Definition 3.1 (Normal domains). We say that $W$ is a normal domain of $Y$ if for any $y \in \partial W$, there exists a countable family of connected, relatively compact, open neighborhoods $\mathcal{U}_{y}=\left\{U_{i}\right\}_{i \in \mathbf{N}}$ such that:

1. For every $i \in \mathbf{N}, \bar{U}_{i+1} \subsetneq U_{i}$, and $\bigcap_{i \in \mathbf{N}} U_{i}=\{y\}$.

2. For any neighborhood $U \in \mathcal{U}_{y}, U \cap W$ is connected.

3. For every smooth Riemannian metric $\rho$ on $U_{0}$, and every $i>1$, the metrics $\delta_{\rho}^{U_{i}}$ and $\delta_{\rho}^{U_{i} \cap W}$ are bi-Lipschitz equivalent on $U_{i} \cap W$. Namely, there exists a real $k_{i} \geq 1$ such that for any $y_{1}, y_{2} \in U_{i} \cap W$

$$
\delta_{\rho}^{U_{i}}\left(y_{1}, y_{2}\right) \leq \delta_{\rho}^{U_{i} \cap W}\left(y_{1}, y_{2}\right) \leq k_{i} \delta_{\rho}^{U_{i}}\left(y_{1}, y_{2}\right)
$$

The distances $\delta_{\rho}^{U_{i}}$ and $\delta_{\rho}^{U_{i} \cap W}$ are defined as follows

$\delta_{\rho}^{U_{i}}\left(y_{1}, y_{2}\right)=\inf \left\{\int_{0}^{1} \sqrt{\rho\left(\gamma^{\prime}(t), \gamma^{\prime}(t)\right)} d t \mid \gamma \in C^{1}\left([0,1] ; U_{i}\right), \gamma(0)=y_{1}, \gamma(1)=y_{2}\right\}$

$\delta_{\rho}^{U_{i} \cap W}\left(y_{1}, y_{2}\right)=\inf \left\{\int_{0}^{1} \sqrt{\rho\left(\gamma^{\prime}(t), \gamma^{\prime}(t)\right)} d t \mid \gamma \in C^{1}\left([0,1] ; U_{i} \cap W\right), \gamma(0)=y_{1}, \gamma(1)=y_{2}\right\}$

Remark 3.2. The property for $W$ to be a normal domain does not depend on the choice of the smooth Riemannian metric $\rho$ on $U_{0}$, because two such Riemannian metrics define bi-Lipschitz equivalent distances on each $U_{i}$ (resp. each $\left.U_{i} \cap W\right), i \geq 1$. Thus, to show that $W \subset Y$ is a normal domain, it is enough to check that the previous conditions are satisfied for just one smooth Riemannian metric on $U_{0}$.

The following lemma exhibits two interesting examples of normal domains.

Lemma 3.3. Let $Y$ be a smooth manifold, and $W \subsetneq Y$ a proper open subset. If the topological boundary $\partial W$ has Hausdorff dimension $<n-1$, or if $\bar{W}$ is a topological submanifold with boundary of $Y$ such that $\partial W$ is locally the graph of a Lipschitz function, then $W$ is a normal domain of $Y$. 
Proof: We assume first that the Hausdorff dimension of $\partial W$ is $<n-1$. The problem is local, thus considering $x \in \partial W$ and a chart around $x$, we can assume that $Y$ is an open subset $V$ of $\mathbf{R}^{n}$ containing 0 , and $W \subsetneq V$ is a proper open subset with $0 \in \partial W$. Let us consider $B_{r} \subset V$ the Euclidean ball of center 0 and radius $r$, and let us take $x_{0}$ and $y_{0}$ in $B_{r} \cap W$. We denote by $W_{x_{0}}$ (resp. $W_{y_{0}}$ ) the set of points in $B_{r} \cap W$ which can be joined to $x_{0}$ (resp. $y_{0}$ ) by a line segment included in $B_{r} \cap W$. Let $B$ be a small closed Euclidean ball centered at $x_{0}$ such that $B \cap \partial W=\emptyset$. There is a well defined radial projection $\pi_{r}: B_{r} \backslash B \rightarrow \partial B$ defined as follows: if $z \in B_{r} \backslash B$, then $\pi_{r}(z)$ is the intersection of the line segment joining $z$ to $x_{0}$ with $\partial B$. The map $\pi_{r}$ is Lipschitz, so that $\pi_{r}\left(B_{r} \cap \partial W\right)$ has Hausdorff dimension $<n-1$ in $\partial B$, hence its complement is dense in $\partial B$. As a consequence, $W_{x_{0}}$ is dense in $B_{r}$, and it is not difficult to check that $W_{x_{0}}$ is also open. The set $W_{y_{0}}$ shares the same properties, so that $W_{x_{0}} \cap W_{y_{0}}$ is dense in $B_{r} \cap W$. As a first consequence $W \cap B_{r}$ is connected. Moreover, we can find a sequence $\hat{z}_{k} \in W_{x_{0}} \cap W_{y_{0}}$ which tends to $y_{0}$. The line segment $\left[x_{0}, z_{k}\right] \subset B_{r}$ tends to the line segment $\left[x_{0}, y_{0}\right] \subset B_{r}$, and the line segment $\left[z_{k}, y_{0}\right] \subset B_{r}$ tends to a point. Therefore, we obtain a sequence of broken line segments included in $B_{r} \cap W$, whose length tends to $d\left(x_{0}, y_{0}\right)$, the Euclidean distance between $x_{0}$ and $y_{0}$. We thus have $\delta_{\rho}^{B_{r}}=\delta_{\rho}^{B_{r} \cap W}$ on $B_{r} \cap W$, where $\rho$ is the Euclidean metric. We see that the neighborhoods $U_{i}=B_{\frac{1}{i+1}}, i \in \mathbf{N}$, satisfy the definition 3.1, for $k_{i}=1$. By remark 3.2, the open subset $W$ is thus normal.

Assume now that $W$ is bounded by a locally Lipschitz hypersurface. As above, looking at a suitable chart around $x \in \partial W$, we can restrict our study to a neighborhood of $0, U=]-\epsilon,+\epsilon\left[{ }^{n} \subset \mathbf{R}^{n}\right.$. We see $\mathbf{R}^{n}$ as the product $\mathbf{R}^{n-1} \times \mathbf{R}$, and the boundary $\partial W \cap U$ is given by a $k$-Lipschitz map $\phi: U^{n-1} \rightarrow \mathbf{R}$, where $\left.U^{n-1}=\right]-\epsilon,+\epsilon\left[^{n-1}\right.$. Restricting to a suitably small neighborhood of $x$, we will assume that the $(z, t) \in U \cap W$ are characterized by $t>\phi(z)$, so that $U \cap W$ is connected. We endow $U$ with the Euclidean metric $\rho$. Since $\phi$ is $k$-Lipschitz, one has

$$
\delta_{\rho}^{U \cap \hat{M}} \leq \sqrt{1+k^{2}} \delta_{\rho}^{U}
$$

The basis of neighborhoods $]-\frac{\epsilon}{i+1},+\frac{\epsilon}{i+1}\left[{ }^{n}\right.$, endowed with the Euclidean metric, satisfies definition 3.1, if we put $k_{i}=k$. By remark $3.2, W$ is a normal domain.

$\diamond$ 


\subsection{The Cauchy boundary of a Kleinian manifold}

We keep the notations of section 3.1. Let $\Omega \subsetneq \mathbf{X}$ be a normal domain, and $M:=\Gamma \backslash \Omega$ a Kleinian manifold, where $\Gamma \subset G$ is a discrete subgroup acting freely properly and discontinuously on $\Omega$. The choice of a basis $X_{1}, \ldots, X_{m}$ on the Lie algebra $\mathfrak{g}$ defines Riemannian metrics $\rho^{G}$ and $\rho^{\Omega}$ on $G$ and $\hat{\Omega}$ respectively. The metric $\rho^{\Omega}$ is the restriction of $\rho^{G}$ to $\hat{\Omega}$. The metric $\rho^{G}$ (resp. $\rho^{\Omega}$ ) is invariant by the left-action of $\Gamma$ on $G$ (resp. on $\hat{\Omega}$ ), hence it induces a metric $\rho^{G_{\Gamma}}$ (resp. $\left.\rho^{M}\right)$ on $G_{\Gamma}$ (resp. on $\hat{M}$ ). Observe that the metric $\rho^{M}$ is precisely the one constructed in section 2.1 for the Cartan geometry $\left(M, \hat{M}, \omega^{M}\right)$, using the basis $X_{1}, \ldots, X_{m}$. To the metrics $\rho^{G_{\Gamma}}$ on $G_{\Gamma}$ and $\rho^{M}$ on $\hat{M}$, we associate the distances $d_{G_{\Gamma}}$ and $d_{M}$, as explained in section 2.1. After observing that the projection $\pi_{\Gamma}:\left(G, \rho^{G}\right) \rightarrow\left(G_{\Gamma}, \rho^{G_{\Gamma}}\right)$ is a Riemannian covering, we get that every connected component of $G_{\Gamma}$ is complete, when endowed with $\rho^{G_{\Gamma}}$. It then follows that the metric space $\left(G_{\Gamma}, d_{G_{\Gamma}}\right)$ is complete.

Lemma 3.4. Let $\Omega \subsetneq \mathbf{X}$ be a normal domain and $M:=\Gamma \backslash \Omega$ a Kleinian manifold. Then a sequence of $\hat{M}$ converges in $G_{\Gamma}$ if and only if it is a Cauchy sequence for $d_{M}$. In particular $\partial \hat{M}$, the topological boundary of $\hat{M}$ in $G_{\Gamma}$, is naturally identified with the Cauchy boundary $\partial_{c} \hat{M}$.

Proof: As we just mentioned, the metric space $\left(G_{\Gamma}, d_{G_{\Gamma}}\right)$ is complete. Observe that whenever $\hat{x}$ and $\hat{y}$ are in a same connected component of $\hat{M}$, $d_{M}(\hat{x}, \hat{y}) \geq d_{G_{\Gamma}}(\hat{x}, \hat{y})$, so that every Cauchy sequence of $\hat{M}$ for $d_{M}$ is a Cauchy sequence for $d_{G_{\Gamma}}$. Hence, every Cauchy sequence of $\hat{M}$ for $d_{M}$ converges in $G_{\Gamma}$.

Conversely, consider a sequence $\left(\hat{x}_{k}\right)$ of $\hat{M}$ converging to $\hat{x}$ in $G_{\Gamma}$. If $\hat{x} \in \hat{M}$, then $\left(\hat{x}_{k}\right)$ is clearly Cauchy for $d_{M}$. Suppose now that $\hat{x} \in \partial \hat{M}$. Because $G \rightarrow \mathbf{X}$ is a fiber bundle, it follows quite easily that $\hat{\Omega}$ is a normal domain of $G$. The projection from $G$ to $G_{\Gamma}$ is a local isometry (with respect to the metrics $\rho^{G}$ and $\rho^{G_{\Gamma}}$ ), so that $\hat{M}$ is a normal domain of $G_{\Gamma}$. In particular, there exists a connected neighborhood $U_{\hat{x}}$ such that $U_{\hat{x}} \cap \hat{M}$ is connected, and a real number $k_{\hat{x}} \geq 1$ satisfying:

$$
\delta_{G_{\Gamma}}^{U_{\hat{x}}}\left(\hat{x}_{1}, \hat{x}_{2}\right) \leq \delta_{G_{\Gamma}}^{U_{\hat{x}} \cap \hat{M}}\left(\hat{x}_{1}, \hat{x}_{2}\right) \leq k_{\hat{x}} \delta_{G_{\Gamma}}^{U_{\hat{x}}}\left(\hat{x}_{1}, \hat{x}_{2}\right)
$$

Recall that $\delta_{G_{\Gamma}}^{U_{\hat{x}} \cap \hat{M}}$ is the distance induced by $\rho^{G_{\Gamma}}$ (or equivalently by $\rho^{M}$ ) on $U_{\hat{x}} \cap \hat{M}$. The inequality (1) shows that $\left(\hat{x}_{k}\right)$, which is a Cauchy sequence for $\delta_{G_{\Gamma}}^{U_{\hat{x}}}$, is also a Cauchy sequence for $\delta_{G_{\Gamma}}^{U_{\hat{x}} \cap \hat{M}}$, hence for $d_{M}$, because $d_{M} \leq$ $\delta_{G_{\Gamma}}^{U_{\hat{x}} \cap \hat{M}}$ on $U_{\hat{x}} \cap \hat{M}$. This completes the proof of the lemma. $\diamond$ 
Remark 3.5. From the previous discussion, it is clear that whenever $M=$ $\Gamma \backslash \mathbf{X}$, then $\partial_{c} M=\emptyset$.

Remark 3.6. When $\Gamma$ is trivial and $M=\Omega$ is a normal domain of $\mathbf{X}$, then lemma 3.4 implies that $\partial_{c} M$ is homeomorphic to $\partial \Omega$. For example, if we consider the Riemannian conformal structure of the Euclidean space $\mathbf{R}^{n}$, then its Cauchy boundary is a single point, because $\mathbf{R}^{n}$ is conformally the model space $\mathbf{X}=\mathbf{S}^{n}$ with a point removed. The reader will check that actually, the Cauchy conformal boundary of any complete flat manifold is a single point. With the same remark, the Cauchy boundary of the conformal structure of the n-dimensional real hyperbolic space is homeomorphic to a sphere of dimension $n-1$.

\section{The boundary map of a geometric immersion}

Let us consider $\left(M, \hat{M}, \omega^{M}\right)$ and $\left(N, \hat{N}, \omega^{N}\right)$ two smooth Cartan geometries modelled on a same model space $\mathbf{X}=G / P$ (notice that $M$ and $N$ automatically have the same dimension, which is that of $\mathbf{X}$ ). We saw in the previous sections how to associate two Cauchy boundaries $\partial_{c} M$ and $\partial_{c} N$ to those structures. In all this section, we consider a geometric immersion $\sigma:\left(M, \hat{M}, \omega^{M}\right) \rightarrow\left(N, \hat{N}, \omega^{N}\right)$, and $s: M \rightarrow N$ the smooth immersion induced by $\sigma$. Our aim is to construct a boundary map $\partial s$ for $s$, defined on the Cauchy boundary $\partial_{c} M$, or at least part of it.

\subsection{Definition of the boundary map}

The construction described in section 2.1 , using a same fixed basis $\left(X_{1}, \ldots, X_{m}\right)$ of $\mathfrak{g}$ yields two Riemannian metrics $\rho^{M}$ and $\rho^{N}$ on $\hat{M}$ and $\hat{N}$ respectively. Because $\sigma^{*}\left(\omega^{N}\right)=\omega^{M}$, the construction implies that $\sigma^{*}\left(\rho^{N}\right)=\rho^{M}$, namely $\sigma$ is an isometric immersion from $\left(\hat{M}, \rho^{M}\right)$ into $\left(\hat{N}, \rho^{N}\right)$. We define a distance $d_{N}^{\sigma}$ on $\sigma(\hat{M})$ in the following way. If $\hat{x}$ and $\hat{y}$ are two points in a same connected component of $\sigma(\hat{M})$, then $d_{N}^{\sigma}(\hat{x}, \hat{y}):=\frac{\delta_{N}^{\sigma}(\hat{x}, \hat{y})}{1+\delta_{N}^{\sigma}(\hat{x}, \hat{y})}$, where $\delta_{N}^{\sigma}(\hat{x}, \hat{y})$ is the infimum of the $\rho^{N}$-lengths of $C^{1}$-curves joining $\hat{x}$ and $\hat{y}$ inside $\sigma(\hat{M})$. If $\hat{x}$ and $\hat{y}$ are in $\sigma(\hat{M})$, but do not stay in a same component of $\sigma(\hat{M})$, we put $d_{N}^{\sigma}(\hat{x}, \hat{y})=2$.

Fact 4.1. The map $\sigma:\left(\hat{M}, d_{M}\right) \rightarrow\left(\sigma(\hat{M}), d_{N}^{\sigma}\right)$ is 1-Lipschitz and a local isometry. When $\sigma$ is not merely an immersion but a geometric embedding, it is a global isometry. 
We always have $d_{N}^{\sigma} \geq d_{N}$, so that $\sigma:\left(\hat{M}, d_{M}\right) \rightarrow\left(\hat{N}, d_{N}\right)$ is also 1-Lipschitz. As a consequence, it extends uniquely to a 1-Lipschitz map $\bar{\sigma}:\left(\hat{M}_{c}, \bar{d}_{M}\right) \rightarrow$ $\left(\hat{N}_{c}, \bar{d}_{N}\right)$. The restriction of $\bar{\sigma}$ to $\partial_{c} \hat{M}$ is called the boundary map of $\sigma$, and we will denote it by $\partial \sigma$.

Since $\sigma$ is equivariant for the action of $P$ on $\hat{M}$ and $\hat{N}$, the map $\bar{\sigma}$ remains equivariant for the action of $P$ on $\hat{M}_{c}$ and $\hat{N}_{c}$. So we also get a map $\bar{s}$ : $M_{c} \rightarrow N_{c}$, and a boundary map $\partial s: \partial_{c} M \rightarrow N_{c}$, but since, as we already mentionned, $M_{c}$ and $\partial_{c} M$ might not be Hausdorff, those maps are maybe not so useful. That is why we are going to restrict our attention to part of the Cauchy boundaries $\partial_{c} \hat{M}$ and $\partial_{c} M$.

Definition 4.2 (Regular set). Let $\sigma:\left(M, \hat{M}, \omega^{M}\right) \rightarrow\left(N, \hat{N}, \omega^{N}\right)$ be a geometric immersion. The regular set of $\partial_{c} \hat{M}$, associated to $\sigma$, is defined as

$$
\hat{\Lambda}_{c}:=\partial \sigma^{-1}(\hat{N})
$$

As a consequence of the $P$-equivariance of $\bar{\sigma}$, the set $\hat{\Lambda}_{c}$ is $P$-invariant and we define $\Lambda_{c}=\hat{\Lambda}_{c} / P$.

\subsection{Behavior of the boundary map on the regular set}

The importance of the set $\Lambda_{c}$ is made clear in proposition 4.4 below. Before stating the proposition, we need one more definition. In section 2.2 , we introduced the notion of points of the first kind in $\partial_{c} \hat{M}$. We now define which ones are "singular", and which are not.

Definition 4.3 (Singular points of the first kind). Let $\left(M, \hat{M}, \omega^{M}\right)$ be a Cartan geometry modelled on $\mathbf{X}=G / P$, and let $\partial_{c} \hat{M}$ be its Cauchy boundary. A point $\hat{z} \in \partial_{c} \hat{M}$ of the first kind is said to be singular if there exist $\hat{x}$ and $\hat{x}^{\prime}$ two distinct points in $\hat{M}, X \in \mathfrak{g}$ and $a>0$, such that:

1. $\alpha(t):=\exp (\hat{x}, t X)$ and $\beta(t):=\exp \left(\hat{x}^{\prime}, t X\right)$ are defined on $[0, a[$.

2. $\lim _{t \rightarrow a^{-}} \alpha(t)=\lim _{t \rightarrow a^{-}} \beta(t)=\hat{z}$.

In the statement, and until the end of the paper, $\partial_{\sigma} \hat{M}$ (resp. $\partial_{s} M$ ) will denote the topological boundary of $\sigma(\hat{M})$ in $\hat{N}$ (resp. of $s(M)$ in $N$ ).

Proposition 4.4. Let us assume that the geometric immersion $\sigma$ is strict (namely, is not onto). Then:

1. The set $\hat{\Lambda}_{c}$ is a nonempty open subset of $\partial_{c} \hat{M}$, which always contains points of the first kind. Moreover, if $\sigma$ is an embedding, no point of the first kind contained in $\hat{\Lambda}_{c}$ is singular. 
2. The map $\bar{\sigma}: \hat{\Lambda}_{c} \cup \hat{M} \rightarrow \hat{N}$ is P-equivariant and continuous.

3. The group $P$ acts freely and properly on $\hat{\Lambda}_{c} \cup \hat{M}$; in particular $\Lambda_{c} \cup M \subset$ $M_{c}$ is Hausdorff.

4. The maps $\partial \sigma$ and $\bar{\sigma}$ induce continuous maps $\partial s: \Lambda_{c} \rightarrow N$ (called the boundary map of $s$ ) and $\bar{s}: \Lambda_{c} \cup M \rightarrow N$.

5. If $\sigma$ is a geometric embedding, then $\partial s$ maps $\Lambda_{c}$ in $\partial_{s} M$ (resp. $\partial \sigma$ maps $\hat{\Lambda}_{c}$ in $\left.\partial_{\sigma} \hat{M}\right)$. Moreover, the image $\partial s\left(\Lambda_{c}\right)$ (resp. $\left.\partial \sigma\left(\hat{\Lambda}_{c}\right)\right)$ contains every accessible point of $\partial_{s} M$ (resp. of $\left.\partial_{\sigma} \hat{M}\right)$, and in particular is dense in $\partial_{s} M$ (resp. in $\left.\partial_{\sigma} \hat{M}\right)$.

To make the last point of the proposition clear, we have to recall what is an accessible point.

Definition 4.5 (Accessible points). A point $x \in \partial_{s} M$ is said to be accessible if there exists a $C^{1}$ path $\gamma:[0,1] \rightarrow N$ such that $\gamma([0,1[) \subset s(M)$ and $\gamma(1)=x$. A point in $\partial_{\sigma} \hat{M}$ is called accessible if it is in the fiber of an accessible point of $\partial_{s} M$.

It is clear that $\hat{x} \in \partial_{\sigma} \hat{M}$ is accessible if and only if there exists a $C^{1}$ path $\hat{\gamma}:[0,1] \rightarrow \hat{N}$ such that $\hat{\gamma}([0,1[) \subset \sigma(\hat{M})$ and $\hat{\gamma}(1)=\hat{x}$. The fact that whenever $\partial_{s} M$ is nonempty, the set of accessible points is dense in $\partial_{s} M$ is standard (see for example [Mo] p.9).

Proof of proposition 4.4: we already saw that the extension $\bar{\sigma}:\left(\hat{M}_{c}, \bar{d}_{M}\right) \rightarrow$ $\left(\hat{N}_{c}, \bar{d}_{N}\right)$ is 1-Lipschitz and $P$-equivariant. Hence the same is true for the restriction $\bar{\sigma}: \hat{\Lambda}_{c} \cup \hat{M} \rightarrow \hat{N}$. This shows the second point of the proposition. Let us now prove the first point. Since we assumed that $\sigma$ is strict, $\partial_{\sigma} \hat{M}$ is nonempty. Let us choose $\hat{z}_{\infty}$ accessible in $\partial_{\sigma} \hat{M}$ (such accessible points exist since we just mentioned that they are actually dense). By definition, there is a $C^{1}$ path $\gamma:[0,1] \rightarrow \hat{N}$ such that $\gamma([0,1)) \subset \sigma(\hat{M})$, and $\gamma(1)=\hat{z}_{\infty}$. Let $\hat{x}$ a point of $\hat{M}$ such that $\sigma(\hat{x})=\gamma(0)$. We consider

$$
E:=\{t \in] 0,1[\mid \exists \alpha:[0, t[\rightarrow \hat{M}, \alpha(0)=\hat{x} \text { and } \sigma(\alpha(u))=\gamma(u) \forall u \in[0, t[\}
$$

Because $\sigma$ is a local diffeomorphism, $E$ is nonempty and we call $t_{0}:=\sup E$. Let us consider $\left(t_{k}\right)$ a sequence of $] 0, t_{0}\left[\right.$ converging to $t_{0}$. Because the $\rho^{M_{-}}$ length of $\alpha\left(\left[t_{k}, t_{k+l}\right]\right)$ is equal to the $\rho^{N}$-length of $\gamma\left(\left[t_{k}, t_{k+l}\right]\right)$, we get that $\alpha\left(t_{k}\right)$ is a Cauchy sequence for $d_{M}$. Hence, $\alpha\left(t_{k}\right)$ converges to $\hat{x}_{\infty}$ in $\hat{M}_{c}$. If $\hat{x}_{\infty}$ is actually in $\hat{M}$, we get a contradiction because the continuity of $\sigma$ 
implies $\sigma\left(\hat{x}_{\infty}\right)=\gamma\left(t_{0}\right)$, and then it would be possible to extend $\alpha$ to a lift of $\gamma$ defined on some $\left[0, t_{0}^{\prime}\right.$ [ with $t_{0}^{\prime}>t_{0}$. We conclude that $\hat{x}_{\infty}$ is in $\partial_{c} \hat{M}$, and by continuity of $\bar{\sigma}, \partial \sigma\left(\hat{x}_{\infty}\right)=\hat{z}_{\infty}$. We conclude that $\hat{\Lambda}_{c}$ is not empty. A Riemannian manifold is always locally complete, so that $\hat{N}$ is open in $\hat{N}_{c}$. Since $\partial \sigma$ is continuous, $\hat{\Lambda}_{c}=\partial \sigma^{-1}(\hat{N})$ is open in $\partial_{c} \hat{N}$.

Let $\hat{z}_{\infty}$ be as above. The exponential map $X \mapsto \exp \left(\hat{z}_{\infty}, X\right)$ is defined on an open subset $\mathcal{W}_{\hat{z}_{\infty}} \in \mathfrak{g}$ containing $0_{\mathfrak{g}}$. There is a neighborhood $\mathcal{V}$ of $0_{\mathfrak{g}}$ in $\mathcal{W}_{\hat{z}_{\infty}}$, which is star-shaped with respect to $0_{\mathfrak{g}}$, on which the exponential map is a diffeomorphism onto its image. Because $\hat{z}_{\infty} \in \partial_{\sigma} \hat{M}$, there exists $Y \in \mathcal{V}$ such that $\hat{z}:=\exp \left(\hat{z}_{\infty}, Y\right)$ is in $\sigma(\hat{M})$. As a consequence $\hat{z}_{\infty}=\exp (\hat{z},-Y)$. Let $\hat{x} \in \hat{M}$ such that $\sigma(\hat{x})=\hat{z}$. Let us define

$$
a:=\sup \{t \in] 0, \infty[, \exp (\hat{x},-t Y) \text { is defined }\}
$$

Notice that if $a<\infty$, the limit $\hat{x}_{\infty}:=\lim _{t \rightarrow a^{-}} \exp (\hat{x},-t Y)$ exists because for any sequence $\left(t_{k}\right)$ converging to $a,\left(\exp \left(\hat{x},-t_{k} Y\right)\right)$ is Cauchy in $\hat{M}$. Moreover $\hat{x}_{\infty}$ is in $\partial_{c} M$, and by definition, it is of the first kind. Now, because we have the relation $\sigma(\exp (\hat{x},-t Y))=\exp (\hat{z},-t Y)$ whenever both terms make sense, we must have $a \leq 1$. By continuity of $\bar{\sigma}, \partial \sigma\left(\hat{x}_{\infty}\right)=\exp (\hat{z},-a Y)$. We infer that $\hat{x}_{\infty} \in \hat{\Lambda}_{c}$, proving that $\hat{\Lambda}_{c}$ contains points of the first kind.

Let us now assume that $\hat{x}_{\infty} \in \hat{\Lambda}_{c}$ is a point of the first kind which is singular: there exist a nonzero $X \in \mathfrak{g}$, distinct points $\hat{x}$ and $\hat{x}^{\prime}$ in $\hat{M}$, such that $t \mapsto \exp (\hat{x}, t X)$ and $t \mapsto \exp \left(\hat{x}^{\prime}, t X\right)$ are defined on $[0, a[$, with $\lim _{t \rightarrow a^{-}} \exp (\hat{x}, t X)=\hat{x}_{\infty}=\lim _{t \rightarrow a^{-}} \exp \left(\hat{x}^{\prime}, t X\right)$. Recall (see section 2.2) that for every $t \in[0, a[, \exp (\sigma(\hat{x}), t X)$ is well defined and

$$
\sigma(\exp (\hat{x}, t X))=\exp (\sigma(\hat{x}), t X)
$$

In particular $\alpha(t):=\exp (\sigma(\hat{x}), t X)$ converges to $\hat{z}_{\infty}:=\partial \sigma\left(\hat{x}_{\infty}\right)$ as $t$ tends to $a$.

For the same reason, the curve $\beta: t \mapsto \exp \left(\sigma\left(\hat{x}^{\prime}\right), t X\right)$, which is actually $t \mapsto \sigma\left(\exp \left(\hat{x}^{\prime}, t X\right)\right)$, is defined on $\left[0, a\left[\right.\right.$, and $\lim _{t \rightarrow a^{-}} \beta(t)=\hat{x}_{\infty}$. If we assume moreover that $\sigma$ is an embedding then $\sigma(\hat{x}) \neq \sigma\left(\hat{x}^{\prime}\right)$. The curves $\alpha$ and $\beta$ are two integral curves of the $\omega^{N}$-constant vector field $\hat{X}$ defined by $\omega^{N}(\hat{X})=X$. Both $\alpha$ and $\beta$ are defined on $[0, a[$, and they satisfy $\alpha(0) \neq \beta(0)$, while $\lim _{t \rightarrow a^{-}} \alpha(t)=\lim _{t \rightarrow a^{-}} \beta(t)=\hat{z}_{\infty}$. The $\omega^{N}$-constant vector field $\hat{X}$ should then vanish at $\hat{z}_{\infty}$. Because $X$ is nonzero and $\omega_{\hat{z}_{\infty}}^{N}: T_{\hat{z}_{\infty}} \hat{N} \rightarrow \mathfrak{g}$ is an isomorphism, we get a contradiction, finishing the proof of the first point.

We now prove the third point. Consider $\left(\hat{x}_{k}\right)$ a sequence of $\hat{\Lambda}_{c} \cup \hat{M}$ converging to $\hat{x}_{\infty} \in \hat{\Lambda}_{c} \cup \hat{M}$, and $\left(p_{k}\right)$ a sequence of $P$ such that $\hat{x}_{k} \cdot p_{k}$ converges to 
$\hat{y}_{\infty} \in \hat{\Lambda}_{c} \cup \hat{M}$. Then $\bar{\sigma}\left(\hat{x}_{k}\right)$ tends to $\bar{\sigma}\left(\hat{x}_{\infty}\right)$ and $\bar{\sigma}\left(\hat{x}_{k}\right) \cdot p_{k}$ tends to $\bar{\sigma}\left(\hat{y}_{\infty}\right)$. Since $P$ acts properly on $\hat{N}$, the sequence $\left(p_{k}\right)$ is bounded in $P$. This shows that the action of $P$ is proper on $\hat{\Lambda}_{c} \cup \hat{M}$. The action of $P$ is also free on $\hat{\Lambda}_{c} \cup \hat{M}$, because it is free on $\hat{N}$, and if $\hat{x} . p=\hat{x}$, for some $\hat{x} \in \hat{\Lambda}_{c} \cup \hat{M}$ and $p \in P$, then $\bar{\sigma}(\hat{x}) \cdot p=\bar{\sigma}(\hat{x})$.

The fourth point is a straightforward consequence of the second and third points.

It remains to consider the last point. Assuming that $\sigma$ is not only an immersion but an embedding, it follows that whenever we consider a sequence $\left(\hat{x}_{k}\right)$ leaving every compact set of $\hat{M}$ and such that $\sigma\left(\hat{x}_{k}\right)$ converges in $\hat{N}$, the limit must be in $\partial_{\sigma} \hat{M}$. Thus, $\partial \sigma$ maps $\hat{\Lambda}_{c}$ in $\partial_{\sigma} \hat{M}$. To see that every accessible point of $\partial_{\sigma} \hat{M}$ is in the image of $\partial \sigma$, we go back to the begining of the proof. Let $\hat{z}_{\infty}$ be an accessible point of $\partial_{\sigma} \hat{M}$, and $\gamma:[0,1] \rightarrow \hat{N}$ such that $\gamma(1)=\hat{z}_{\infty}$ and $\gamma\left(\left[0,1[) \subset \sigma(\hat{M})\right.\right.$. Then if $\left(t_{k}\right)$ is a sequence of $[0,1[$ tending to 1 , and $\left(\hat{x}_{k}\right)$ is the sequence of $\hat{M}$ such that $\sigma\left(\hat{x}_{k}\right)=\gamma\left(t_{k}\right)$, we saw that $\hat{x}_{k}$ converges to $\hat{x}_{\infty}$ in $\hat{\Lambda}_{c}$ such that $\partial \sigma\left(\hat{x}_{\infty}\right)=\hat{z}_{\infty}$.

By $P$-equivariance, every accessible point of $\partial_{s} M$ is also in the image of $\partial s$. $\diamond$

Remark 4.6. Whenever the metric space $\left(\hat{N}, d_{N}\right)$ is complete, the regular set $\hat{\Lambda}_{c}$ is the whole Cauchy boundary $\partial_{c} \hat{M}$. In this case, the manifold $M$ embeds as an open subset of a Hausdorff space $M_{c}$ (possibly equal to $M$ ), and the geometric immersion extends to a continuous map $\bar{s}: M_{c} \rightarrow N$.

\subsection{Basic maximality criteria}

Proposition 4.4 yields actually sufficient conditions ensuring that a Cartan geometry $\left(M, \hat{M}, \omega^{M}\right)$ is (strongly) geometrically maximal. For instance, the first point of proposition 4.4 implies that if $\partial_{c} M=\emptyset$, then $M$ is strongly maximal. By remark 3.5, if $\Gamma \subset G$ acts freely properly and discontinuously on $\mathbf{X}$, the quotient $\Gamma \backslash \mathbf{X}$ is also strongly geometrically maximal among Cartan geometries modelled on $\mathbf{X}$. As a direct corollary of the first and third points of proposition 4.4, we also get:

Proposition 4.7. Let $\left(M, \hat{M}, \omega^{M}\right)$ be a Cartan geometry modelled on $\mathbf{X}=$ $G / P$.

1. If there is no point of the first kind in $\partial_{c} \hat{M}$, then $\left(M, \hat{M}, \omega^{M}\right)$ is strongly geometrically maximal.

2. If there is no open subset of $\hat{M}_{c}$, properly containing $\hat{M}$, on which the action of $P$ is free and proper, then $\left(M, \hat{M}, \omega^{M}\right)$ is strongly geometrically maximal. 
3. If among the set of points of the first kind, the singular ones are dense, then $\left(M, \hat{M}, \omega^{M}\right)$ is geometrically maximal.

The conditions given in the proposition are difficult to check. Our task in sections 5 and 6 will be to exhibit concrete situations where they are satisfied.

\section{A maximality criterion for Kleinian manifolds}

Thanks to our study of the Cauchy boundary for Kleinian manifolds, and the basic maximality criteria stated above, we are now ready to prove theorem 1.4. After the proof, we will give applications in different geometric contexts.

\subsection{Proof of theorem 1.4}

We assume that we are under the hypotheses of theorem 1.4, namely $\mathbf{X}=$ $G / P$ is a model space, $\Omega \subsetneq \mathbf{X}$ is a normal domain, and $\Gamma \subset G$ acts freely properly and discontinuously on $\Omega$, yielding a Kleinian manifold $M=\Gamma \backslash \Omega$. Moreover, there is no open subset of $\bar{\Omega}:=\partial \Omega \cup \Omega$, properly containing $\Omega$, on which the action of $\Gamma$ is free and proper (recall that $\partial \Omega$ denotes the topological boundary of $\Omega$ in $\mathbf{X}$ ).

We keep the notations of section 3.1. Assume for a contradiction that $\left(\hat{M}, M, \omega^{M}\right)$ is not strongly geometrically maximal: there exists $\left(\hat{N}, N, \omega^{N}\right)$ a Cartan geometry modelled on $\mathbf{X}$ and a geometric immersion $\sigma:\left(\hat{M}, M, \omega^{M}\right) \rightarrow$ $\left(\hat{N}, N, \omega^{N}\right)$ which is not onto. Proposition 4.4 then ensures that the regular set $\hat{\Lambda}_{c}$ is a nonempty open subset of $\partial_{c} \hat{M}$. Lemma 3.4 implies that $\hat{\Lambda}_{c}$ is a nonempty, $P$-invariant, open subset of $\partial \hat{M}$, where $\partial \hat{M}$ is the topological boundary of $\hat{M}$ in $G_{\Gamma}$. Let $\check{\Lambda}$ be the inverse image of $\hat{\Lambda}_{c}$ by the covering map $\pi_{\Gamma}: G \rightarrow G_{\Gamma}$, and $\Lambda:=\pi_{X}(\check{\Lambda})$, where $\pi_{X}: G \rightarrow \mathbf{X}$ is the bundle map. Then, $\Lambda \cup \Omega$ is an open subset of $\bar{\Omega}$, which is $\Gamma$-invariant because $\hat{\Lambda}_{c} \cup \hat{M}$ is $P$-invariant.

Let us prove that the action of $\Gamma$ on $\Lambda \cup \Omega$ is free and proper if and only if the action of $P$ on $\hat{\Lambda}_{c} \cup \hat{M}$ is free and proper. We pick $x \in \Lambda \cup \Omega$, and $\left(x_{k}\right)$ a sequence of $\Lambda \cup \Omega$ converging to $x$. We assume that $\left(\gamma_{k}\right)$ is a sequence of $\Gamma$ such that $\gamma_{k}\left(x_{k}\right)$ converges to some point $y \in \Lambda \cup \Omega$. We lift $\left(x_{k}\right)$ into a sequence $\left(\hat{x}_{k}\right)$ of $\check{\Lambda} \cup \hat{\Omega}$ converging to a point $\hat{x}$. There exists a sequence $\left(p_{k}\right)$ of $P$ such that $\gamma_{k}\left(\hat{x}_{k}\right) \cdot p_{k}$ tends to a point $\hat{y}$. Because the actions of $\Gamma$ and $P$ commute on $\tilde{\Lambda} \cup \hat{\Omega}$, the map $\pi_{\Gamma}$ is $P$-equivariant. We thus get

$$
\pi_{\Gamma}\left(\hat{x}_{k}\right) \cdot p_{k}=\pi_{\Gamma}\left(\gamma_{k}\left(\hat{x}_{k}\right) \cdot p_{k}\right)
$$


where $\pi_{\Gamma}\left(\hat{x}_{k}\right)$ is a sequence converging to $\pi_{\Gamma}(\hat{x}) \in \hat{\Lambda}_{c} \cup \hat{M}$. The actions of $\Gamma$ and $P$ on $G$ are proper, hence because $\left(\hat{x}_{k}\right)$ and $\gamma_{k}\left(\hat{x}_{k}\right) \cdot p_{k}$ are both converging, $\left(\gamma_{k}\right)$ is bounded in $\Gamma$ if and only if $\left(p_{k}\right)$ is bounded in $P$. We get that $\Gamma$ acts properly on $\Lambda \cup \Omega$ if and only if $P$ acts properly on $\hat{\Lambda}_{c} \cup \hat{M}$.

Now, assume that $\left(x_{k}\right)$ is constant to a point $x$, and $\left(\gamma_{k}\right)$ is constant to an element $\gamma$. Then $\left(\hat{x}_{k}\right)$ and $\left(p_{k}\right)$ can be chosen constant to $\hat{x}$ and $p$ respectively. But the actions of $\Gamma$ and $P$ on $G$ are free, so that $\gamma=1_{\Gamma}$ if and only if $p=1_{P}$. We get that $\Gamma$ acts freely on $\Lambda \cup \Omega$ if and only if $P$ acts freely on $\hat{\Lambda}_{c} \cup \hat{M}$.

Proposition 4.4 does ensure that the action of $P$ is free and proper on $\hat{\Lambda}_{c} \cup \hat{M}$. We thus get a contradiction with the fact that $\Gamma$ does not act freely and properly on any open subset of $\bar{\Omega}$ properly containing $\Omega$.

\subsection{Examples illustrating theorem 1.4}

Our aim here is to list some concrete applications of the general theorem 1.4 to classical geometric structures.

We will begin with Riemannian conformal geometry. It has been known since the works of E. Cartan that given a Riemannian structure $(M, g)$ of dimension $n \geq 3$, the conformal class $[g]$ determines a canonical Cartan geometry $\left(M, \hat{M}, \omega^{M}\right)$ modelled on $\mathbf{S}^{n}=P O(1, n+1) / P$ (here $P$ is a parabolic subgroup isometric to the group of conformal transformations of $\mathbf{R}^{n}$.) The bundle $\hat{M}$ can be seen as a sub-bundle of 2-jets of frames on $M$ (see [Ko], [Sh] Chap. 7). If $(M, g)$ and $(N, h)$ are two $n$-dimensional Riemannian manifolds, and if $s: M \rightarrow N$ is a conformal immersion, i.e the map $s$ is smooth and $s^{*} h=e^{\varphi} g$ for some smooth function $\varphi$ on $M$, then $s$ naturally lifts to a bundle immersion $\sigma:\left(M, \hat{M}, \omega^{M}\right) \rightarrow\left(N, \hat{N}, \omega^{N}\right)$ between the associated canonical Cartan geometries. Moreover, $\sigma^{*} \omega^{N}=\omega^{M}$. Hence, $\sigma$ is a geometric immersion in the sense of section 1.2.2.

\subsubsection{Complete flat Riemannian structures: proof of corollary 1.5}

A complete flat structure of dimension $n, n \geq 3$, is a quotient $\Gamma \backslash \Omega$, where $\Omega=\mathbf{S}^{n} \backslash\{p\}$, and $\Gamma$ is a discrete subgroup of $P O(1, n+1)$ fixing $p$. By lemma 3.3, $\Omega$ is a normal domain. As soon as $\Gamma \neq\{i d\}$, the action of $\Gamma$ on $\partial \Omega$ is nowhere free. We can apply theorem 1.4 and deduce immediately corollary 1.5 . 


\subsubsection{Complete hyperbolic manifolds: proof of corollary 1.6}

Such manifolds are quotients $M_{\Gamma}:=\Gamma \backslash \Omega$, where $\Omega$ is the upper hemisphere in $\mathbf{S}^{n}$, and $\Gamma \subset P O(1, n+1)$ is a discrete subgroup acting freely properly and discontinuously on $\Omega$. If $\Lambda_{\Gamma}$ denotes the limit set of $\Gamma$ on $\partial \Omega$, it is well known that $\Omega_{\Gamma}:=\mathbf{S}^{n} \backslash \Lambda_{\Gamma}$ is the biggest open subset of $\mathbf{S}^{n}$ (for the inclusion) on which the action of $\Gamma$ is proper (see [Ka] sec. 4.6 and 4.7). Hence, theorem 1.4 implies that if $\Lambda_{\Gamma}=\partial \Omega$ then $\Gamma \backslash \mathbf{H}^{n}$ is strongly conformally maximal. Conversely, if $\Lambda_{\Gamma} \neq \partial \Omega$, then $M_{\Gamma}$ embeds conformally as a proper open subset of $\Gamma \backslash \Omega_{\Gamma}$, hence is not conformally maximal. This yields corollary 1.6 .

\subsubsection{Conformal Kleinian manifolds}

Let us go further in the discussion above about hyperbolic manifolds. If $\Gamma$ is a noncompact discrete subgroup of $P O(1, n+1)$, then the action of $\Gamma$ is free and proper on no open set properly containing $\Omega_{\Gamma}$. A direct consequence of theorem 1.4 is that whenever $\Omega_{\Gamma}$ is a normal domain, and when $n \geq 3$, a Kleinian manifold $\Gamma \backslash \Omega_{\Gamma}$ is strongly conformally maximal. Of course, it is not easy to determine for which Kleinian groups $\Gamma$, the domain of discontinuity $\Omega_{\Gamma}$ is normal.

\subsubsection{Conformally maximal Lorentzian manifolds}

As for the Riemannian case, any conformal Lorentz structure of dimension $\geq 3$ defines a normal Cartan geometry. The $n$-dimensional model space is now $\mathbf{X}=P O(2, n) / P$, where $P$ is the parabolic subgroup stabilizing an isotropic line in $\mathbf{R}^{2, n}$. The space $\mathbf{X}$ is usually called Einstein's universe of dimension $n$, denoted $\mathbf{E i n}_{n}$.

We are going to exhibit a class of Lorentz manifolds which are conformally maximal. The $n$-dimensional anti-de Sitter space, denoted $\mathbf{A d S}_{n}$, is the Lorentzian analogue of the real hyperbolic space. A model for this space is the following: in $\mathbf{R}^{n+2}$ equiped with the quadratic form $q^{2, n}(x)=-x_{1}^{2}-$ $x_{2}^{2}+\ldots+x_{n+2}^{2}$, let us consider $Q_{-1}:=\left\{u \in \mathbf{R}^{n+2} \mid q^{2, n}(u)=-1\right\}$. The anti-de Sitter space is the quadric $Q_{-1}$ endowed with the Lorentz metric induced by $q^{2, n}$. It is a complete Lorentz manifold of constant sectional curvature -1 . The space $\mathbf{A d} \mathbf{S}_{n}$ is not conformally maximal: it embeds conformally as a normal open subset $\Omega \subset \mathbf{E i n}_{n}$. In odd dimension, the group $O(2,2 n+1)$ contains a subgroup isomorphic to $U(1, n)$, which leaves $\mathbf{A d S}_{2 n+1}=\Omega \subset \mathbf{E i n}_{2 n+1}$ invariant. Now, let $\Gamma$ be a lattice in $U(1, n)$. In [Fr2] (see proposition 7, and remark 5), it was shown that the action of such 
a $\Gamma$ is proper on no open subset of $\partial \Omega$. As a consequence of theorem 1.4, we infer:

Corollary 5.1. Any anti-de Sitter manifold $\Gamma \backslash \mathbf{A d} \mathbf{S}_{2 n+1}$, with $\Gamma$ a lattice in $P U(1, n)$, is conformally maximal.

\subsubsection{Maximal $C R$-structures}

The boundary of the $n+1$-dimensional complex hyperbolic space $(n \geq 1)$ is a $2 n+1$-sphere, endowed with a flat strictly pseudoconvex $C R$-structure. Its group of $C R$-automorphisms is $P U(1, n+1)$, so that, from the $C R$ point of view, it is the homogeneous space $\mathbf{X}=P U(1, n+1) / P$ (with $P$ a parabolic subgroup). Actually, any strictly pseudoconvex $C R$-structure on a manifold $M^{2 n+1}$ defines a canonical Cartan geometry $\left(M, \hat{M}, \omega^{M}\right)$ modelled on $\mathbf{X}$ (see $[\mathrm{Ch}],[\mathrm{He} 2],[\mathrm{T}],[\mathrm{CS}]$ ).

There is a subgroup $\operatorname{Heis}(2 n+1) \subset P U(1, n+1)$, isomorphic to the $2 n+1$ dimensional Heisenberg group, which fixes a point $o \in \mathbf{X}$, and acts simply transitively on $\Omega=\mathbf{X} \backslash\{o\}$ ([Go, chap. 4.2]). From this action, Heis $(2 n+1)$ inherits a flat strictly pseudoconvex $C R$-structure, which is moreover leftinvariant. Since $\Omega$ is a normal domain by lemma 3.3, a straightforward consequence of theorem 1.4 is:

Corollary 5.2. Any CR-structure of the form $\Gamma \backslash \operatorname{Heis}(2 n+1), n \geq 1$, with $\Gamma \neq\{i d\}$ a discrete subgroup of Heis $(2 n+1)$, is maximal among all $2 n+1$-dimensional strictly pseudoconvex $C R$ structures.

With the same proof, a similar statement holds for quotients of the $4 n+3$ dimensional quaternionic analogue of the Heisenberg space.

In the same way, the unit tangent bundle to the real hyperbolic space $\mathbf{H}^{n+1}$ $(n \geq 1)$ is endowed with a flat $C R$-structure. Actually, $T^{1} \mathbf{H}^{n+1}$ is an open orbit $\Omega$ of $O(1, n+1) \subset P U(1, n+1)$ in $\mathbf{X}$, the boundary of which is a sphere $\mathbf{S}^{n}$ (see for instance [BS, Proposition 6.1]). The action of $O(1, n+1)$ on $\partial \Omega$ is identified to that on the boundary of $\mathbf{H}^{n+1}$. Because $\partial \Omega$ is a smooth submanifold, $\Omega$ is a normal domain of $\mathbf{X}$ by lemma 3.3. We can use theorem 1.4 and get:

Corollary 5.3. The flat CR-structure on the unit tangent bundle of a complete hyperbolic manifold of finite volume is $C R$-maximal.

\subsubsection{Some projectively maximal structures}

A projective structure on $M$ is the data of a class $\mathcal{C}$ of torsion free affine connexions on $M$, all defining the same geodesics up to reparametriza- 
tion. Given a projective structure on $M$, one can build a canonical Cartan geometry $\left(M, \hat{M}, \omega^{M}\right)$ modelled on the projective space $\mathbf{X}=\mathbf{R} \mathbf{P}^{n}=$ $P G L(n+1, \mathbf{R}) / P$ (with $P$ the stabilizer of a line). A projective immersion between two affine manifolds of the same dimension $\left(M, \nabla_{M}\right)$ and $\left(N, \nabla_{N}\right)$, is an immersion mapping geodesics to geodesics (without necessarily preserving the affine parametrization). Such an immersion lifts to an immersion $\sigma$ between the canonical Cartan bundles $\left(M, \hat{M}, \omega^{M}\right)$ and $\left(N, \hat{N}, \omega^{N}\right)$. Thus, it is a geometrical immersion, as defined in 1.2.2.

The affine flat space $\mathbf{R}^{n}$ embeds projectively as an open subset $\Omega \subset \mathbf{R} \mathbf{P}^{n}$. The boundary $\partial \Omega$ is a codimension one $\mathbf{R} \mathbf{P}^{n-1}$ in $\mathbf{R} \mathbf{P}^{n}$. In particular $\Omega$ is a normal domain thanks to lemma 3.3. Any translation of $\mathbf{R}^{n}$ extends as a projective transformation of $\mathbf{R P}^{n}$, which fixes $\partial \Omega$ pointwise. Applying theorem 1.4, we get:

Corollary 5.4. For $n \geq 3$, any complete flat affine manifold $\Gamma \backslash \mathbf{R}^{n}$, where $\Gamma \subset \operatorname{Aff}(n, \mathbf{R})$ contains a nontrivial translation, is strongly projectively maximal. This is in particular the case for any complete flat Riemannian manifold, except the Euclidean space.

With the same proof as for corollary 1.6, but using this time the projective model for the hyperbolic space, we also get:

Corollary 5.5. For a complete hyperbolic manifold $M=\Gamma \backslash \mathbf{H}^{n}, n \geq 3$, the following conditions are equivalent:

1. $M$ is strongly projectively maximal.

2. The limit set $\Lambda_{\Gamma}$ is equal to $\mathbf{S}^{n-1}=\partial \mathbf{H}^{n}$.

\section{Other maximality criteria}

\subsection{A general result}

Let $\mathbf{X}=G / P$ be a model space, and $\left(M, \hat{M}, \omega^{M}\right)$ a Cartan geometry modelled on $\mathbf{X}$. In proposition 4.4, we saw that whenever the action of $P$ is nowhere proper on $\partial_{c} \hat{M}$, the Cartan geometry $\left(M, \hat{M}, \omega^{M}\right)$ is (strongly) geometrically maximal. We are now going to give conditions ensuring maximality, which involve this time properties of the action of $\operatorname{Aut}\left(\hat{M}, \omega^{M}\right)$ on $\partial_{c} \hat{M}$ (the group $\operatorname{Aut}\left(\hat{M}, \omega^{M}\right)$ has been introduced in 1.2.2). Let us first explain why the action of $\operatorname{Aut}\left(\hat{M}, \omega^{M}\right)$ extends to the boundary $\partial_{c} \hat{M}$. If $\phi$ is an element of $\operatorname{Aut}\left(\hat{M}, \omega^{M}\right)$, then it acts isometrically on $\left(\hat{M}, d_{M}\right)$. The isometry $\phi$ thus extends to an isometry $\bar{\phi}$ of $\left(\hat{M}_{c}, \bar{d}_{M}\right)$. In the sequel, we endow 
$\operatorname{Aut}\left(\hat{M}, \omega^{M}\right)$ with the topology of uniform convergence on compact subsets of $\hat{M}$. For this topology, $\operatorname{Aut}\left(\hat{M}, \omega^{M}\right)$ is a closed subgroup of $\operatorname{Homeo}(\hat{M})$, and the action of $\operatorname{Aut}\left(\hat{M}, \omega^{M}\right)$ on $\hat{M}$ is free and proper. This comes from the fact that $\operatorname{Aut}\left(\hat{M}, \omega^{M}\right)$ preserves a framing on $\hat{M}$. The reader will find details about this in [Ko, Theorem 3.2 and its proof], under the assumption that the manifold supporting the framing is connected. Here, though $M$ is assumed to be connected, $\hat{M}$ is generally not. Nevertheless, the proof carries over to our situation, because the actions of $P$ and $\operatorname{Aut}\left(\hat{M}, \omega^{M}\right)$ commute, and $P$ acts transitively on the set of connected components of $\hat{M}$.

As a last remark, let us mention that the extended action $\operatorname{Aut}\left(\hat{M}, \omega^{M}\right) \times$ $\hat{M}_{c} \rightarrow \hat{M}_{c}$ is continuous.

Proposition 6.1. Let $\left(M, \hat{M}, \omega^{M}\right)$ be a Cartan geometry modelled on the space $\mathbf{X}=G / P$.

1. Assume that for every point $\hat{z}_{\infty}$ of the first kind in $\partial_{c} \hat{M}$, there exists a sequence $\left(\phi_{k}\right)$ in $\operatorname{Aut}\left(\hat{M}, \omega^{M}\right)$ which does not tend to the identity map, and such that $\bar{d}_{M}\left(\hat{z}_{\infty}, \phi_{k}\left(\hat{z}_{\infty}\right)\right)$ tends to 0 . Then $\left(M, \hat{M}, \omega^{M}\right)$ is geometrically maximal.

2. In particular, if every point of the first kind in $\partial_{c} \hat{M}$ has a nontrivial stabilizer in Aut $\left(\hat{M}, \omega^{M}\right)$, then $\left(M, \hat{M}, \omega^{M}\right)$ is geometrically maximal.

Proof: We only prove the first point, which immediately implies the second. Let us assume, for a contradiction, that there is a strict geometric embedding $\sigma:\left(M, \hat{M}, \omega^{M}\right) \rightarrow\left(N, \hat{N}, \omega^{N}\right)$, where $\left(N, \hat{N}, \omega^{N}\right)$ is a Cartan geometry modelled on $\mathbf{X}$. By proposition 4.4, the regular set $\hat{\Lambda}_{c}$ associated to $\sigma$ is a nonempty open subset of $\partial_{c} \hat{M}$ and $\sigma$ extends to a continuous map $\bar{\sigma}: \hat{\Lambda}_{c} \cup \hat{M} \rightarrow \hat{N}$. Again by proposition 4.4, the set $\hat{\Lambda}_{c}$ contains points of the first kind. We pick $\hat{z}_{\infty} \in \hat{\Lambda}_{c}$ such a point, and call $\hat{y}_{\infty}:=$ $\bar{\sigma}\left(\hat{z}_{\infty}\right)$. Given the hypotheses on the sequence $\left(\phi_{k}\right)$, and replacing $\left(\phi_{k}\right)$ by a subsequence if necessary, we may assume that either $\phi_{k}$ converges to $\phi_{\infty} \neq 1$ in $\operatorname{Aut}\left(\hat{M}, \omega^{M}\right)$, or $\left(\phi_{k}\right)$ leaves every compact set of $\operatorname{Aut}\left(\hat{M}, \omega^{M}\right)$.

Let us assume we are in the case where $\phi_{k}$ converges to $\phi_{\infty} \neq 1$ in $\operatorname{Aut}\left(\hat{M}, \omega^{M}\right)$. Because $\bar{\phi}_{k}$ and $\bar{\phi}_{\infty}$ act isometrically on $\left(\hat{M}_{c}, \bar{d}_{M}\right)$, it is easily checked that $\bar{\phi}_{k}$ converges pointwise to $\bar{\phi}_{\infty}$ on $\hat{M}_{c}$. In particular $\bar{\phi}_{\infty}\left(\hat{z}_{\infty}\right)=\hat{z}_{\infty}$. The point $\hat{z}_{\infty}$ is of the first kind, hence there exists a nonzero $X \in \mathfrak{g}$, and $\hat{x} \in \hat{M}$, such that $t \mapsto \exp (\hat{x}, t X)$ is defined on $\left[0, a\left[\right.\right.$, and $\lim _{t \rightarrow a^{-}} \exp (\hat{x}, t X)=\hat{z}_{\infty}$. Recall (see section 2.2) that for every $t \in\left[0, a\left[, \exp \left(\phi_{\infty}(\hat{x}), t X\right)\right.\right.$ is well defined and

$$
\phi_{\infty}(\exp (\hat{x}, t X))=\exp \left(\phi_{\infty}(\hat{x}), t X\right)
$$


In particular $\exp \left(\phi_{\infty}(\hat{x}), t X\right)$ converges to $\hat{z}_{\infty}$ as $t$ tends to $a$. Recall that $\operatorname{Aut}\left(\hat{M}, \omega^{M}\right)$ acts freely on $\hat{M}$, so that $\phi_{\infty}(\hat{x}) \neq \hat{x}$. We get that the point of the first kind $\hat{z}_{\infty}$ is singular, contradicting the first point of proposition 4.4.

We now assume that the sequence $\left(\phi_{k}\right)$ leaves every compact subset of $\operatorname{Aut}\left(\hat{M}, \omega^{M}\right)$. The Riemannian metrics $\rho^{M}$ and $\rho^{N}$ define two volume forms $\mathrm{Vol}^{M}$ and $\mathrm{Vol}^{N}$ on $\hat{M}$ and $\hat{N}$ respectively. Because $\sigma$ is a geometric embedding, $\sigma^{*}\left(\mathrm{Vol}^{N}\right)=\mathrm{Vol}^{M}$. We choose $\epsilon_{0}>0$ and denote by $B_{1}$ (resp. $\left.B_{2}\right)$ the $\bar{d}_{M}$-ball of center $\hat{z}_{\infty}$ and radius $\epsilon_{0}$ in $\hat{M}_{c}$ (resp. the $d_{N}$-ball of center $\hat{y}_{\infty}$ and radius $\epsilon_{0}$ in $\hat{N}$ ). If we chose $\epsilon_{0}$ small enough, then $B_{1} \subset \hat{\Lambda}_{c} \cup \hat{M}$ (recall that by proposition 4.4, $\hat{\Lambda}_{c} \cup \hat{M}$ is open in $\hat{M}_{c}$ ), and $B_{2}$ has finite volume for $\mathrm{Vol}^{N}$. Now, $\bar{\sigma}$ is a 1-Lipschitz map so that $\sigma\left(B_{1} \cap \hat{M}\right) \subset B_{2}$. Since $\sigma$ preserves the volume, $B_{1} \cap \hat{M}$ has finite volume for $\mathrm{Vol}^{M}$. Let $B_{1}^{\prime}$ be the $\bar{d}_{M}$-ball of center $\hat{z}_{\infty}$ and radius $\frac{\epsilon_{0}}{2}$. Let $U$ be a small open subset of $B_{1}^{\prime} \cap \hat{M}$, which is relatively compact in $B_{1}^{\prime} \cap \hat{M}$. We already mentioned that the action of $\operatorname{Aut}\left(\hat{M}, \omega^{M}\right)$ is proper on $\hat{M}$. Hence, considering a subsequence of $\left(\phi_{k}\right)$ if necessary, we may assume that $\phi_{m}(U) \cap \phi_{n}(U)=\emptyset$ for every integers $m, n$ with $m \neq n$. Because $\bar{\phi}_{k}$ acts isometrically for $\bar{d}_{M}$, and because $\bar{d}_{M}\left(\hat{z}_{\infty}, \bar{\phi}_{k}\left(\hat{z}_{\infty}\right)\right)$ tends to 0 , we have $\phi_{k}(U) \subset B_{1} \cap \hat{M}$ for every $k \geq k_{0}$ big enough. But $\operatorname{Aut}\left(\hat{M}, \omega^{M}\right)$ also preserves $\operatorname{Vol}^{M}$, so that $\phi_{m}(U) \cap \phi_{n}(U)=\emptyset$ for $m \neq n$ greater that $k_{0}$ implies that $B_{1} \cap \hat{M}$ has infinite volume for $\operatorname{Vol}^{M}$ : contradiction. $\diamond$

\subsection{Cartan geometries and coverings}

Before going further, let us recall some basic facts about Cartan geometries and coverings. Assume that $\left(M, \hat{M}, \omega^{M}\right)$ is a Cartan geometry modelled on X. Let $\rho: \mathcal{M} \rightarrow M$ be a covering map. Then the $P$-principal bundle $\hat{M}$ can be lifted into a $P$-principal bundle $\hat{\mathcal{M}}$ over $\mathcal{M}$, and the 1 -form $\omega^{M}$ can also be lifted into a 1 -form $\omega^{\mathcal{M}}$ on $\hat{\mathcal{M}}$. The triple $\left(\mathcal{M}, \hat{\mathcal{M}}, \omega^{\mathcal{M}}\right)$ is a Cartan geometry modelled on $\mathbf{X}$, that is called the lift to $\mathcal{M}$ of the Cartan geometry on $M$. We get a commutative diagram

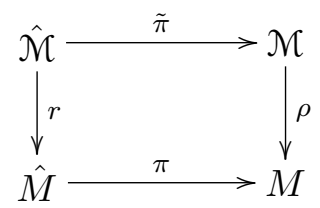

where $\tilde{\pi}$ and $\pi$ are the bundle maps, and $\rho$ and $r$ are covering maps. The map $r$ is $P$-equivariant, and by definition of $\omega^{\mathcal{M}}$, one has $r^{*}\left(\omega^{M}\right)=\omega^{\mathcal{M}}$. If 
$\rho$ is a regular covering map, induced by the action of a group $\Gamma$ on $\mathcal{M}$, then the action of $\Gamma$ lifts to $\hat{\mathcal{M}}$, and this is an action by automorphism of $\left(\hat{\mathcal{M}}, \omega^{\mathcal{M}}\right)$ which induces the covering map $r$.

\section{Holonomy morphism}

Of particular importance in the sequel will be the case where $M \subsetneq \mathbf{X}$ is a proper open subset of $\mathbf{X}$, endowed with its canonical flat Cartan geometry modelled on $\mathbf{X}$. We keep the notations introduced above.

Lemma 6.2. Let $M \subset \mathbf{X}$ be a proper open subset, and $\rho: \mathcal{M} \rightarrow M$ its universal cover. There exists an epimorphism hol : Aut $\left(\hat{\mathcal{M}}, \omega^{\mathcal{M}}\right) \rightarrow$ $\operatorname{Aut}\left(\hat{M}, \omega^{M}\right)$, called holonomy morphism, satisfying for every $\varphi \in \operatorname{Aut}\left(\hat{\mathcal{M}}, \omega^{\mathcal{M}}\right)$ the equivariance relation

$$
r \circ \varphi=\operatorname{hol}(\varphi) \circ r
$$

The pair $(r, \mathbf{h o l})$ is the translation at the level of the Cartan bundle, of the classical pair (developping map, holonomy morphism) for $(G, \mathbf{X})$-structures (see [Th, 3.3, 3.4]).

Proof: Let us recall the following classical fact, that we will call Liouville's theorem in the sequel. If $U$ and $V$ are two connected open subsets of the Lie group $G$, and $\varphi: U \rightarrow V$ is an immersion satisfying $\varphi^{*} \omega^{G}=\omega^{G}$, then $\varphi$ is the restriction to $U$ of the left multiplication by an element $g_{\varphi} \in G$. Indeed, there exists $g_{\varphi}$ such that $g \mapsto g_{\varphi}^{-1} \cdot \varphi(g)$ fixes a point of $U$. Because $\left(g_{\varphi}^{-1} \cdot \varphi\right)^{*} \omega^{G}=\omega^{G}$, the map $g_{\varphi}^{-1} \cdot \varphi$ leaves invariant a framing on $U$. It is then classical that $g_{\varphi}^{-1} \cdot \varphi$ must be the identical map on $U$. The geometry $\left(\mathcal{M}, \hat{\mathcal{M}}, \omega^{\mathcal{M}}\right)$ is flat, hence locally isomorphic to $\left(\mathbf{X}, G, \omega^{G}\right)$. As a consequence of Liouville's theorem, if $\varphi$ is an element of $\operatorname{Aut}\left(\hat{\mathcal{M}}, \omega^{\mathcal{M N}}\right)$, and if $U$ is a small connected open subset of $\hat{\mathcal{M}}$, there must exist an element $g_{\varphi} \in G$ such that $r \circ \varphi=g_{\varphi} \circ r$ on $U$. Now, the set of points in $\hat{\mathcal{M}}$ where the relation $r \circ \varphi=g_{\varphi} \circ r$ holds is clearly closed. Liouville's theorem ensures that it is also open, so that $r \circ \varphi=g_{\varphi} \circ r$ holds on a connected component of $\hat{\mathcal{H}}$. Because $r$ is $P$-equivariant and $P$ acts transitively on the set of connected components of $\hat{\mathcal{M}}$, the relation eventually holds on $\hat{\mathcal{M}}$. It is not hard to see, applying again Liouville's theorem, that $\varphi \mapsto g_{\varphi}$ is actually a group homomorphism. We define $\operatorname{hol}(\varphi):=g_{\varphi}$. Since $r$ is onto, the relation $r \circ \varphi=\operatorname{hol}(\varphi) \circ r$ shows that hol maps actually $\operatorname{Aut}\left(\hat{\mathcal{M}}, \omega^{\mathcal{M}}\right)$ in $\operatorname{Aut}\left(\hat{M}, \omega^{M}\right)$. Because $\mathcal{M}$ is simply connected, every element of $\operatorname{Aut}\left(\hat{M}, \omega^{M}\right)$ can be lifted to an element of $\operatorname{Aut}\left(\hat{\mathcal{M}}, \omega^{\mathcal{M}}\right)$, so that hol is an epimorphism. $\diamond$ 


\subsection{A maximality criterion for coverings}

In theorem 1.4 we gave a condition for a quotient of some open subset of the model space $\mathbf{X}$ to be geometrically maximal. This section will be devoted to the "opposite" question. If $M \subsetneq \mathbf{X}$ is a proper open subset, when is a nontrivial covering of $M$ maximal among the Cartan geometries modelled on $\mathbf{X}$ ?

In theorem 1.4, we needed an assumption of "normality" on the Kleinian manifold we considered. Here, we will need a condition of another kind on the domain $M \subsetneq \mathbf{X}$, that we introduce below.

Definition 6.3 (Domains with property $(H)$ ). Let $Y$ be a smooth manifold, $W \subsetneq Y$ a proper, nonempty, connected open subset, and $\partial W$ the topological boundary of $W$ in $Y$. We endow $Y$ with a Riemannian metric $g$. The open set $W$ is said to have property $(H)$ if for every $x_{\infty} \in \partial W$, and every sequence $\left(x_{k}\right)$ of $W$ converging to $x_{\infty}$, there exists a sequence of $C^{1}$-loops $\alpha_{k}$ in $W$ such that:

1. For every $k \in \mathbf{N}, \alpha_{k}$ is based at $x_{k}$, and is homotopically nontrivial in $W$.

2. The g-length of $\alpha_{k}$ tends to zero as $k$ tends to infinity.

For instance, the space $\mathbf{R}^{n}, n \geq 2$, with finitely many disjoint codimension 2 affine subspaces removed has property $(H)$. It is of course possible to build much more complicated examples.

We can now state our main result about maximality of coverings.

Theorem 6.4. Let $\mathbf{X}=G / P$ be a model space, and $M \subsetneq \mathbf{X}$ be an open subset having property $(H)$. Let $\mathcal{M}$ be the universal cover of $M$, that we endow with its natural flat Cartan geometry $\left(\mathcal{M}, \hat{\mathcal{M}}, \omega^{\mathcal{M}}\right)$ modelled on $\mathbf{X}$. Then $\left(\mathcal{M}, \hat{\mathcal{N}}, \omega^{\mathcal{N}}\right)$ is geometrically maximal.

Proof: We use the notations $\rho, r, \pi, \tilde{\pi}$ of section 6.2 , and denote by $\left(M, \hat{M}, \omega^{M}\right)$ the flat canonical Cartan geometry of the Kleinian manifold $M \subset \mathbf{X}$ (see section 3.1). As explained in 6.2, this Cartan geometry lifts to a flat Car$\tan$ geometry $\left(\mathcal{M}, \hat{\mathcal{M}}, \omega^{\mathcal{M}}\right)$ on the universal cover $\mathcal{M}$. The covering map $\rho: \mathcal{M} \rightarrow M$ is regular, and induced by the action of $\pi_{1}(M)$ on $\mathcal{M}$. The action of $\pi_{1}(M)$ lifts into an action by automorphisms of $\hat{\mathcal{M}}$, yielding the covering map $r: \hat{\mathcal{M}} \rightarrow \hat{M}$. The map $r$ is a geometric covering from $\left(\hat{\mathcal{M}}, \omega^{\mathcal{M}}\right)$ to $\left(\hat{M}, \omega^{M}\right)$, and in particular a 1-Lipschitz map from $\left(\hat{\mathcal{M}}, d_{\mathcal{M}}\right)$ to $\left(G, d_{G}\right)$, 
this latter metric space being complete. As a consequence, the map $r$ extends to a 1-Lipschitz map $\bar{r}:\left(\hat{\mathcal{M}}_{c}, \bar{d}_{\mathcal{N}}\right) \rightarrow\left(G, d_{G}\right)$. The restriction of $\bar{r}$ to $\partial_{c} \hat{\mathcal{M}}$ is denoted by $\partial r$.

Lemma 6.5. The map $\partial r$ maps $\partial_{c} \hat{\mathcal{M}}$ to $\partial \hat{M}$, the topological boundary of $\hat{M}$ in $G$.

Proof: Let $\hat{z}_{\infty} \in \partial_{c} \hat{\mathcal{M}}$, and $\hat{x}_{\infty}:=\partial r\left(\hat{z}_{\infty}\right)$. The point $\hat{x}_{\infty}$ is clearly in $\hat{M} \cup \partial \hat{M}$. We want to show that $\hat{x}_{\infty} \notin \hat{M}$. Assume the contrary. Then there is a small compact neighborhood $K$ of $\hat{x}_{\infty}$ in $\hat{M}$, such that the inverse image of $K$ by $r$ is a disjoint union $\bigcup_{\gamma \in \pi_{1}(M)} \gamma(\tilde{K})$, with $\tilde{K}$ compact in $\hat{\mathcal{M}}$. Because the action of $\pi_{1}(M)$ on $\hat{\mathcal{N}}$ is by automorphisms, $\pi_{1}(M)$ acts isometrically for the distance $d_{\mathcal{M}}$. In particular, the action of $\pi_{1}(M)$ is proper on $\hat{\mathcal{M}}$ and there must exist $C>0$ such that $\inf _{\gamma \in \pi_{1}(M), \gamma \neq 1} d_{\mathcal{M}}(\tilde{K}, \gamma(\tilde{K})) \geq C$, or in other words $d_{\mathcal{M}}\left(\gamma(\tilde{K}), \gamma^{\prime}(\tilde{K})\right) \geq C$ for every $\gamma \neq \gamma^{\prime} \in \pi_{1}(M)$. Let $\left(\hat{z}_{k}\right)$ be a sequence of $\hat{\mathcal{M}}$ converging to $\hat{z}_{\infty}$ (the convergence being in the metric space $\left.\left(\hat{\mathcal{M}}_{c}, \bar{d}_{\mathcal{M}}\right)\right)$. Because $\left(\hat{z}_{k}\right)$ is a Cauchy sequence for $d_{\mathcal{M}}$, we must have that for $k$ big enough, all the $\hat{z}_{k}$ are in a single $\gamma_{0}(\tilde{K})$. Thus $\hat{z}_{\infty} \in \gamma_{0}(\tilde{K})$, contradicting $\hat{z}_{\infty} \in \partial_{c} \hat{\mathcal{M}} . \diamond$

We pick $\hat{z}_{\infty}$ a point of the first kind in $\partial_{c} \hat{\mathcal{M}}$. Such points exist, because $\hat{M}$, as a proper open subset of $G$, contains incomplete $\omega^{G}$-constant curves (and in particular $\omega^{M}$-constant curves), which lift into incomplete $\omega^{\mathcal{M}_{\text {-constant }}}$ curves in $\hat{\mathcal{M}}$. There exists a nonzero $X \in \mathfrak{g}$, and $\hat{z} \in \hat{\mathcal{M}}$, such that $\hat{\beta}$ : $t \mapsto \exp (\hat{z}, t X)$ is defined on $\left[0, a\left[\right.\right.$ and $\lim _{t \rightarrow a^{-}} \hat{\beta}(t)=\hat{z}_{\infty}$. For $t \in[0, a[$, we define $\hat{\alpha}(t):=r(\hat{\beta}(t))=\exp (r(\hat{z}), t X)$. The limit, as $t$ tends to $a$ of $\hat{\alpha}(t)$ is $\hat{x}_{\infty}:=\partial r\left(\hat{z}_{\infty}\right)$, which by lemma 6.5 is a point of $\partial \hat{M}$. In particular, $\lim _{t \rightarrow a^{-}} \pi(\alpha(t))$ exists and is a point $x_{\infty} \in \partial M$. Let $\left(t_{k}\right)$ be a sequence of $[0, a$ [ tending to $a$. Because $M$ has property $(H)$, there exists for each $k$ a loop $\alpha_{k}:[0,1] \rightarrow M$, which is based at $\alpha\left(t_{k}\right)$, and is homotopically nontrivial in $M$. Moreover for any fixed Riemannian metric on $\mathbf{X}$, the length of $\alpha_{k}$ tends to 0 as $k \rightarrow \infty$. Let $U$ be a small open neighborhood of $x_{\infty}$ in $\mathbf{X}$, so that $\pi^{-1}(U)$ is trivialized as a product $U \times P$, and $\hat{x}_{\infty}$ corresponds to the point $\left(x_{\infty}, 1_{P}\right)$. We lift $\alpha_{k}$ into the loop $\hat{\alpha}_{k}(t):=\left(\alpha_{k}(t), 1_{P}\right), t \in[0,1]$. Because $r$ is a covering map, we can lift $\hat{\alpha}_{k}$ into a path $\hat{\beta}_{k}:[0,1] \rightarrow \hat{\mathcal{M}}$, such that $\hat{\beta}_{k}(0)=\hat{\beta}\left(t_{k}\right)$. The first observation is that $\hat{\beta}_{k}$ is not a loop in $\hat{\mathcal{M}}$. Indeed, the relation $\rho \circ \tilde{\pi}=\pi \circ r$ shows that $\tilde{\pi}\left(\hat{\beta}_{k}\right)$ is a lift of $\alpha_{k}$ in $\mathcal{M}$. But $\alpha_{k}$ being homotopically nontrivial in $M$, no lift of $\alpha_{k}$ in $\mathcal{M}$ is a loop. Hence, for each $k \in \mathbf{N}$, there exists $\gamma_{k} \in \pi_{1}(M), \gamma_{k} \neq 1$, such that $\hat{\beta}_{k}(1)=\gamma_{k} \cdot \hat{\beta}_{k}(0)=\gamma_{k} \cdot \hat{\beta}\left(t_{k}\right)$. 
The second observation is that for any Riemannian metric on $G$, the length of $\hat{\alpha}_{k}$ tends to 0 . In particular, the $\rho^{M}$-length of $\hat{\alpha}_{k}$ has this property. Since the $\rho^{\mathcal{M}}$-length of $\hat{\beta}_{k}$ is the $\rho^{M}$-length of $\hat{\alpha}_{k}$, we have $\lim _{k \rightarrow \infty} d_{\mathcal{M}}\left(\hat{\beta}_{k}(1), \hat{\beta}\left(t_{k}\right)\right)=$ 0 , which yields

$$
\lim _{k \rightarrow \infty} d_{\mathcal{M}}\left(\gamma_{k} \cdot \hat{\beta}\left(t_{k}\right), \hat{\beta}\left(t_{k}\right)\right)=0
$$

The element $\gamma_{k}$ is an isometry of $\left(\hat{\mathcal{M}}, d_{\mathcal{M}}\right)$, hence extends to an isometry $\bar{\gamma}_{k}$ of $\left(\hat{\mathcal{M}}_{c}, \bar{d}_{\mathcal{M}}\right)$. Since $\hat{\beta}\left(t_{k}\right)$ tends to $\hat{z}_{\infty}$, we infer from equation (3) that $\lim _{k \rightarrow \infty} \bar{d}_{\mathcal{N}}\left(\gamma_{k} . \hat{z}_{\infty}, \hat{z}_{\infty}\right)=0$. After noticing that $\pi_{1}(M)$ is discrete, and $\gamma_{k} \neq 1$ for every $k$, hence the sequence $\left(\gamma_{k}\right)$ does not converge to 1 , we can use proposition 6.1 and conclude that $\left(\mathcal{M}, \hat{\mathcal{N}}, \omega^{\mathcal{M}}\right)$ is geometrically maximal. $\diamond$

In the spirit of section 5.2, the reader is invited to test the theorem above in different explicit geometric situations. Let us just emphasize the interesting feature that given $M \subset \mathbf{X}$, having property $(H)$ does not depend on the geometric structure we put on $\mathbf{X}$. For instance, if $M$ is $\mathbf{R}^{n}$ with an affine subspace of codimension 2 removed, then $M$, seen as an open subset of $\mathbf{R}^{n}$ has property $(H)$. From $\mathbf{R}^{n}, M$ inherits a flat (incomplete) Riemannian metric, a flat affine connexion, a flat pseudo-Riemannian metric of type $(p, q), 1 \leq p \leq q$. Each of these structures can be lifted to the universal cover $\mathbf{R}^{n}$ of $M$, and theorem 6.4 ensures that all the lifted structures are maximal in their category.

From the proof of theorem 6.4, we can deduce the following corollary, the first point of which will be repeatedly used in sections 8 and 9 for proving theorems 1.1 and 9.1 .

Corollary 6.6. Let $\mathbf{X}=G / P$ be a model space, $M \subsetneq \mathbf{X}$ be an open subset with $\pi_{1}(M)$ isomorphic to $\mathbf{Z}$. We assume that $M$ satisfies the following stronger version of property $(H)$ : for every $x_{\infty} \in \partial M$, and every sequence $\left(x_{k}\right)$ of $M$ converging to $x_{\infty}$, there exists a sequence of $C^{1}$-loops $\alpha_{k}$ such that $\alpha_{k}$ generates $\pi_{1}\left(M, x_{k}\right)$ and the length of $\alpha_{k}$ tends to 0 as $k$ tends to infinity.

1. Every flat Cartan geometry $\left(M^{\prime}, \hat{M}^{\prime}, \omega^{M^{\prime}}\right)$ modelled on $\mathbf{X}$, the universal cover of which is $\left(\mathcal{M}, \hat{\mathcal{M}}, \omega^{\mathcal{M}}\right)$, and which is not geometrically isomorphic to the flat Cartan geometry of a quotient $\Gamma \backslash M$, for $\Gamma \subset G$ acting freely properly and discontinuously on $M$, is geometrically maximal.

2. In particular, if $\operatorname{Aut}\left(M, \omega^{M}\right)$ is trivial, then any nontrivial covering of $M$ is geometrically maximal. 
Proof: Let us see the implications of our assumption about the open set $M \subsetneq \mathbf{X}$. Let $\gamma_{*}$ be a generator of $\pi_{1}(M)$, that we see as an element of $\operatorname{Aut}\left(\hat{\mathcal{M}}, \omega^{\mathcal{M}}\right)$. The open set $M$ satisfies property $(H)$, so the proof of theorem 6.4 still holds. Let us look at the part of the proof just after Lemma 6.5, and let us keep the notations introduced there. The sequence of loops $\alpha_{k}$ is now chosen under our standing assumptions, namely $\alpha_{k}$ generates $\pi_{1}\left(M, x_{k}\right)$ and the length of $\alpha_{k}$ tends to 0 as $k$ tends to infinity. This implies that the sequence $\left(\gamma_{k}\right)$, on which we had no control in theorem 6.4 , can now be chosen constant, equal to $\gamma_{*}$. We thus get the property that whenever $\hat{z}_{\infty}$ is a point of the first kind in $\partial_{c} \hat{\mathcal{M}}$, then $\gamma_{*} . \hat{z}_{\infty}=\hat{z}_{\infty}$.

We now prove the first point of the corollary. By hypothesis, there exists a discrete subgroup $\Gamma^{\prime} \subset \operatorname{Aut}\left(\hat{\mathcal{M}}, \omega^{\mathcal{M N}}\right)$, such that $\hat{M}^{\prime}=\Gamma^{\prime} \backslash \hat{\mathcal{M}}, M^{\prime}=\Gamma^{\prime} \backslash \mathcal{M}$, and $\omega^{M^{\prime}}$ is the form induced by $\omega^{\mathcal{M}}$ on the quotient $\hat{M}^{\prime}=\Gamma^{\prime} \backslash \hat{\mathcal{M}}$. We call $\pi^{\prime}:\left(\hat{\mathcal{M}}, d_{\mathcal{M}}\right) \rightarrow\left(\hat{M}^{\prime}, d_{M^{\prime}}\right)$, and $\bar{\pi}^{\prime}:\left(\hat{\mathcal{M}}_{c}, \bar{d}_{\mathcal{M}}\right) \rightarrow\left(\mathcal{M}_{c}^{\prime}, \bar{d}_{M^{\prime}}\right)$ the extended 1-Lipschitz map. Assume that $\left(M^{\prime}, \hat{M}^{\prime}, \omega^{M^{\prime}}\right)$ is not geometrically maximal, and consider $\sigma:\left(M^{\prime}, \hat{M}^{\prime}, \omega^{M^{\prime}}\right) \rightarrow\left(N, \hat{N}, \omega^{N}\right)$ a geometric embedding, where $\left(N, \hat{N}, \omega^{N}\right)$ is a Cartan geometry modelled on $\mathbf{X}$. We denote by $\hat{\Lambda}_{c} \subset \partial_{c} \hat{M}^{\prime}$ the regular set associated to $\sigma$.

By proposition 4.4, $\hat{\Lambda}_{c}$ does contain points of the first kind, and none of those points is singular. Let $\hat{x}_{\infty}$ be such a point. There exists $X \in \mathfrak{g}, \hat{x} \in \hat{M}^{\prime}$, such that $\alpha: t \mapsto \exp (\hat{x}, t X)$ is defined on $\left[0, a\left[\right.\right.$ and $\lim _{t \rightarrow a^{-}} \exp (\hat{x}, t X)=\hat{x}_{\infty}$. Let $\hat{z}$ be a point of $\hat{\mathcal{M}}$ such that $\pi^{\prime}(\hat{z})=\hat{x}$. The curve $\hat{\alpha}: t \mapsto \exp (\hat{z}, t X)$ is also defined on $\left[0, a\left[\right.\right.$, and if $\left(t_{k}\right)$ is a sequence of [0,a[ converging to $a$, then the $\rho^{\mathcal{M}}$-length of $\hat{\alpha}$ between $t_{k}$ and $t_{k+1}$ is the $\rho^{M^{\prime}}$-length of $\alpha$ between $t_{k}$ and $t_{k+1}$. It follows that $\hat{\alpha}\left(t_{k}\right)$ is a Cauchy sequence in $\left(\hat{\mathcal{M}}, d_{\mathcal{M}}\right)$ and we conclude that there is a point $\hat{z}_{\infty} \in \partial_{c} \hat{\mathcal{M}}$ such that $\hat{z}_{\infty}=\lim _{t \rightarrow a^{-}} \exp (\hat{z}, t X)$. By definition, $\hat{z}_{\infty}$ is of the first kind, and we must have $\gamma_{*} \cdot \hat{z}_{\infty}=\hat{z}_{\infty}$. We infer that the curve $\hat{\beta}: t \mapsto \exp \left(\gamma_{*} . \hat{z}, t X\right)$ which is actually $\gamma_{*} . \hat{\alpha}$ is defined on $[0, a[$ and satisfies $\lim _{t \rightarrow a^{-}} \hat{\beta}(t)=\hat{z}_{\infty}$. Calling $\beta(t):=\pi^{\prime}(\hat{\beta}(t))=\exp \left(\pi^{\prime}\left(\gamma_{*} . \hat{z}\right), t X\right)$, we get $\lim _{t \rightarrow a^{-}} \beta(t)=\hat{x}_{\infty}$. Since the point $\hat{x}_{\infty}$ is nonsingular, we must have $\pi^{\prime}\left(\gamma_{*} . \hat{z}\right)=\hat{x}$. In other words, there exists $\gamma^{\prime} \in \Gamma^{\prime}$ such that $\gamma^{\prime} . \hat{z}=\gamma_{*} . \hat{z}$, and because $\operatorname{Aut}\left(\hat{\mathcal{M}}, \omega^{\mathcal{M}}\right)$ acts freely on $\hat{\mathcal{M}}$, we get $\gamma^{\prime}=\gamma_{*}$, hence $\pi_{1}(M) \subset \Gamma^{\prime}$. Now, $\pi_{1}(M)$ is the kernel of the holonomy morphism hol associated to the covering $\rho: \hat{M} \rightarrow M$. In particular $\pi_{1}(M)$ is normal in $\operatorname{Aut}\left(\hat{\mathcal{M}}, \omega^{\mathcal{M}}\right)$, hence in $\Gamma^{\prime}$. The group $\Gamma:=\Gamma^{\prime} / \pi_{1}(M)$ thus acts freely properly discontinuously on $M$ by automorphisms, and by Liouville's theorem, $\Gamma$ is a subgroup of $G$.

We now show the second point of the corollary. By the first point, it is enough to check that $\left(M, \hat{M}, \omega^{M}\right)$ is never geometrically equivalent to one of its nontrivial covers. Because $\pi_{1}(M)$ is the kernel of the holonomy mor- 
phism hol : $\operatorname{Aut}\left(\hat{\mathcal{M}}, \omega^{\mathcal{M}}\right) \rightarrow \operatorname{Aut}\left(\hat{M}, \omega^{M}\right)$, our assumption that $\operatorname{Aut}\left(\hat{M}, \omega^{M}\right)$ is trivial implies $\pi_{1}(M)=\operatorname{Aut}\left(\hat{\mathcal{N}}, \omega^{\mathcal{M}}\right)$. Hence, the assertion boils down to the remark that no proper subgroup of $\pi_{1}(M)$ is conjugated, into $\pi_{1}(M)$, to $\pi_{1}(M)$ itself. $\diamond$

\section{Rank one homogeneous parabolic geometries are maximal or flat}

We are now entering the second part of the paper, leading to the proofs of theorems 1.1 and 9.1. A key step towards those theorems is to show that any noncompact Riemannian conformal structure (resp. $C R$-structure) which is homogeneous but not maximal has to be conformally flat (resp. $C R$-flat). Actually, proposition 7.1 below provides a unified proof of this result for a wider class of geometric structures called rank one parabolic geometries. Those are the Cartan geometries $\left(M, \hat{M}, \omega^{M}\right)$ modelled on the spaces $\mathbf{X}=G / P$, where $G$ is a simple, noncompact Lie group of real rank one, and $P$ is a parabolic subgroup of $G$. Those spaces $\mathbf{X}$ are the Hadamard boundary of the hyperbolic spaces $\partial \mathbf{H}_{\mathbf{K}}^{d}$, where $\mathbf{K}$ can be the reals $\mathbf{R}$, the complex numbers $\mathbf{C}$, the quaternions $\mathbf{H}$ or the octonions $\mathbf{O}$. We will assume $d \geq 4$ when $\mathbf{K}=\mathbf{R}$ and $d \geq 2$ in the other cases (observe that when $\mathbf{K}=\mathbf{O}$, $d$ must be equal to 2 ). Let us recall the different Lie groups $G$ involved:

- $G=S O(1, d), d \geq 4$ for $\mathbf{K}=\mathbf{R}$. The space $\mathbf{X}=\partial \mathbf{H}_{\mathbf{R}}^{d}$ is a sphere $\mathbf{S}^{d-1}$.

- $G=S U(1, d), d \geq 2$ for $\mathbf{K}=\mathbf{C}$. The space $\mathbf{X}=\partial \mathbf{H}_{\mathbf{C}}^{d}$ is a sphere $\mathbf{S}^{2 d-1}$.

- $G=S p(1, d), d \geq 2$ for $\mathbf{K}=\mathbf{H}$. The space $\mathbf{X}=\partial \mathbf{H}_{\mathbf{H}}^{d}$ is a sphere $\mathbf{S}^{4 d-1}$.

- $G=F_{4}^{-20}$ if $\mathbf{K}=\mathbf{O}$. The space $\mathbf{X}=\partial \mathbf{H}_{\mathbf{O}}^{2}$ is a sphere $\mathbf{S}^{15}$.

All what we need about the structure of the Lie algebras $\mathfrak{g}$ is listed in the few lines below (see for instance the reference $[\mathrm{Kn}]$ ). The Cartan subalgebra $\mathfrak{a}$ is 1-dimensional, and its adjoint action defines a splitting $\mathfrak{g}=$ $\mathfrak{g}_{-2} \oplus \mathfrak{g}_{-1} \oplus \mathfrak{a} \oplus \mathfrak{k} \oplus \mathfrak{g}_{+1} \oplus \mathfrak{g}_{+2}$, with $\mathfrak{g}_{-2}=\mathfrak{g}_{+2}=0$ when $\mathbf{K}=\mathbf{R}$. The parabolic subgroup $P$ is a semi-direct product $(A \times K) \ltimes N^{+}$, where $A, K$ and $N^{+}$have Lie algebra $\mathfrak{a}, \mathfrak{k}$ and $\mathfrak{g}_{+1} \oplus \mathfrak{g}_{+2}$ respectively. The groups $A$ and $N^{+}$are connected, and $K$ is compact. There is a group isomorphism $\lambda: A \rightarrow \mathbf{R}_{+}^{*}$ such that, defining $\mathfrak{g}_{0}:=\mathfrak{a} \oplus \mathfrak{k}$, the property Ad $(a) \cdot \xi=\lambda^{j}(a) \xi$ characterizes $\xi \in \mathfrak{g}_{j}, j \in\{-2, \ldots,+2\}$.

We will restrict our study to rank one parabolic geometries $\left(M, \hat{M}, \omega^{M}\right)$ which are regular, namely the curvature function $\kappa: \hat{M} \rightarrow \operatorname{Hom}\left(\Lambda^{2}(\mathfrak{g} / \mathfrak{p})^{*}, \mathfrak{g}\right)$ introduced in 1.2.1, satisfies the condition that for every $\hat{x} \in \hat{M}, \kappa_{\hat{x}}\left(\overline{\mathfrak{g}}_{i}, \overline{\mathfrak{g}}_{j}\right) \subset$ $\mathfrak{g}_{i+j+1}$, where $i, j \in\{-2,-1\}$, and $\overline{\mathfrak{g}}_{i}$ is the projection of $\mathfrak{g}_{i}$ on $\mathfrak{g} / \mathfrak{p}$. Ob- 
serve that conformal Riemannian structures, strictly pseudoconvex $C R$ structures, as well as contact quaternionic and octonionic structures as defined by $\mathrm{O}$. Biquard in [Bi], canonically define a rank one parabolic geometry such that $\omega^{M}$ is regular (see also [CS]). The proposition below thus applies to all these structures.

Proposition 7.1. Let $\left(M, \hat{M}, \omega^{M}\right)$ be a regular, rank one parabolic geometry modelled on $\mathbf{X}=\partial \mathbf{H}_{\mathbf{K}}^{d}$. If $\left(M, \hat{M}, \omega^{M}\right)$ is homogeneous, but not geometrically maximal among all regular Cartan geometries modelled on $\mathbf{X}$, it is flat.

Proof: The basic idea is to build on $M$ a Riemannian metric, which may vanish at some points, but is not identically zero as soon as $M$ is not flat, and which is invariant by the pseudogroup of local automorphisms. For instance, when $(M,[g])$ is a Riemannian conformal structure of dimension $\geq 4$, the metric $\|W\|_{g} g$ (where $\|W\|_{g}$ stands for the $g$-norm of the Weyl tensor) has this property. It seems that this technique goes back to $[\mathrm{H}]$, and is used in $[\mathrm{Ob}]$ in the same way as we do in theorem 7.3 below (see also [Web] for the $C R$-case). We thank the referee for drawing our attention to those references, and for suggesting us how to use them in the present framework. The following lemma generalizes these ideas to all regular, rank one parabolic geometries.

Lemma 7.2. Let $\left(N, \hat{N}, \omega^{N}\right)$ be a regular, rank one parabolic geometry. Let $F \subset N$ be the closed subset where the Cartan curvature vanishes, and assume $F \neq N$. Then there is a finite family $N_{1}, \ldots, N_{s}$ of nonempty, pairwise disjoint, open subsets of $N \backslash F$, and on each $N_{j}$ a smooth Riemannian metric $g_{j}$, as well as a smooth volume form $\beta_{j}$, with the following properties:

1. The union $\bigcup_{j=1}^{s} N_{j}$ is dense in $N \backslash F$.

2. Seen as a section from $N_{j}$ into $\operatorname{Sym}^{2}(T N)$, the metric $g_{j}$ extends continuously to $\bar{N}_{j}$, the closure of $N_{j}$ in $N$.

3. The volume form $\beta_{j}$ extends to a continuous $n$-form $\tilde{\beta}_{j}$ on $N$.

4. If $\varphi: U \rightarrow V$ is a local automorphism between two open subsets of $N$, then for $j=1, \ldots, s, \varphi$ maps $U \cap N_{j}$ to $V \cap N_{j}$ and satisfies $\varphi^{*} g_{j}=g_{j}$, $\varphi^{*} \beta_{j}=\beta_{j}$.

Proof: Let us define $V:=\operatorname{Hom}\left(\Lambda^{2}(\mathfrak{g} / \mathfrak{p})^{*}, \mathfrak{g}\right)$. Recall that the right action of $P$ on $V$ is given by the formula: $(\alpha . p)(\xi, \zeta):=\operatorname{Ad} p^{-1} \cdot \alpha(\operatorname{Ad} p . \xi$, Ad $p . \zeta)$, 
for every $\alpha \in V, \xi, \zeta \in \mathfrak{g} / \mathfrak{p}$ and $p \in P$. Because $\kappa$ is $P$-equivariant, it vanishes at a point $\hat{x}$ in the fiber of $x \in M$ if and only if it vanishes on all the fiber of $x$. Thus, it makes sense to speak about the closed set $F \subset N$ where the Cartan curvature vanishes. From the decomposition of $\mathfrak{g}$ into eigenspaces for the adjoint action of $A$, we get a splitting of he $P$-module $V$ as a direct sum $V_{0} \oplus V_{1} \oplus \ldots \oplus V_{6}$. An element $\alpha$ is in $V_{j}$ if and only if for every $a \in A, \alpha . a=\lambda^{-j}(a)$. $\alpha$. The regularity condition on the curvature $\kappa$ implies that $\kappa\left(\overline{\mathfrak{g}}_{-1}, \overline{\mathfrak{g}}_{-1}\right) \subset \mathfrak{g}_{-1} \oplus \mathfrak{g}_{0} \oplus \mathfrak{g}_{1} \oplus \mathfrak{g}_{2}$. As a consequence, the curvature $\kappa$ takes actually values in $V_{1} \oplus \ldots \oplus V_{6}$.

Let us denote by $\hat{F}$ the inverse image of $F$ in $\hat{N}$. For every $\hat{x} \in \hat{N} \backslash \hat{F}$, define $i(\hat{x}):=\min \left\{j \in\{1, \ldots 6\} \mid \kappa_{\hat{x}}^{(j)} \neq 0\right\}$. Since for any $j \in\{1, \ldots, 6\}$, and any $p \in P, V_{j}$ is mapped by $p$ to $V_{j} \oplus V_{j+1} \oplus \ldots \oplus V_{6}$, and since $\kappa$ is a $P$-equivariant map, the integer $i(\hat{x})$ is constant along the fibers of $\hat{N} \backslash \hat{F}$. We get a well-defined map $i: N \backslash F \rightarrow\{1, \ldots, 6\}$, and we define

$$
N_{j}:=\{x \in N \backslash F \mid i(x)=j \text { on a neighborhood of } x\}
$$

Under the assumption $F \subsetneq N$, there exists a nonempty $N_{j}$. We discard the empty ones and get a new labelling $N_{1}, \ldots, N_{s}$ for some $s \in\{1, \ldots, 6\}$. Clearly, the $N_{j}$ 's are pairwise disjoint open subsets and $\bigcup_{j=1}^{s} N_{j}$ is dense in $N \backslash F$. We observe also that if $\varphi: U \rightarrow V$ is a local automorphism between two open subsets of $N$, then $i(\varphi(x))=i(x)$ for every $x \in U \backslash F$, so that for $j=1, \ldots, s, \varphi$ maps $U \cap N_{j}$ to $V \cap N_{j}$.

Let $x \in N, u \in T_{x} N, \hat{x} \in \hat{N}$ and $\hat{u} \in T_{\hat{x}} \hat{N}$ such that $D_{\hat{x}} \pi(\hat{u})=u$. Define $\iota_{\hat{x}}(u)$ as the projection on $\mathfrak{g} / \mathfrak{p}$ of $\omega_{\hat{x}}^{N}(\hat{u})$. This does not depend on the choice of $\hat{u}$, and yields an isomorphism $\iota_{\hat{x}}: T_{x} M \rightarrow \mathfrak{g} / \mathfrak{p}$, satifying the equivariance relation: $\iota_{\hat{x} . p}=\operatorname{Ad} p^{-1} \cdot \iota_{\hat{x}}$. The splitting $\mathfrak{g} / \mathfrak{p}=\overline{\mathfrak{g}}_{-2} \oplus \overline{\mathfrak{g}}_{-1}$ allows to write $\iota_{\hat{x}}=\iota_{\hat{x}}^{(-2)}+\iota_{\hat{x}}^{(-1)}$.

We choose on $\mathfrak{g} / \mathfrak{p}$ a $K$-invariant scalar product $<,>$, for which $\overline{\mathfrak{g}}_{-2}$ and $\overline{\mathfrak{g}}_{-1}$ are orthogonal. This is possible since $K$ is compact and the adjoint action of $K$ preserves the splitting $\mathfrak{g} / \mathfrak{p}=\overline{\mathfrak{g}}_{-2} \oplus \overline{\mathfrak{g}}_{-1}$. We denote by $\|$.$\| the norm$ defined by $<,>$. We also consider $\left(\xi_{1}, \ldots, \xi_{n}\right)$ an orthonormal basis for $<,>$, and assume that $\xi_{1}, \ldots \xi_{m}$ spans $\overline{\mathfrak{g}}_{-2}$, while $\xi_{m+1}, \ldots, \xi_{n}$ spans $\overline{\mathfrak{g}}_{-1}$. We then define $\beta:=\xi_{1}^{*} \wedge \ldots \wedge \xi_{n}^{*}$. Finally, for $j=1, \ldots, s$, we consider on $\hat{N}$ the continuous function $f_{j}(\hat{x}):=\left\|\kappa_{\hat{x}}^{(j)}\right\|^{\frac{1}{j}}$, where $\kappa_{\hat{x}}^{(j)}$ denotes the component of $\kappa_{\hat{x}}$ on $V_{j}$.

When $\hat{x}$ is in $\hat{N}_{j}$, the inverse image of $N_{j}$ in $\hat{N}$, we have the relation $f_{j}(\hat{x} . l)=$ $f_{j}(\hat{x})$ if $l \in K \ltimes N^{+}$and $f_{j}(\hat{x} . a)=\lambda^{-1}(a) f_{j}(\hat{x})$ if $a \in A$, while $(\operatorname{Ad} l)^{*} \beta=\beta$ and $(\operatorname{Ad} a)^{*} \beta=\lambda^{m+n}(a) \beta$. 
Hence, for every $u, v \in T_{x} N$, and any $n$-uple $\left(u_{1}, \ldots, u_{n}\right)$ of $T_{x} N$, the expressions

$$
g_{j}(u, v):=f_{j}(\hat{x})^{4}<\iota_{\hat{x}}^{(-2)}(u), \iota_{\hat{x}}^{(-2)}(v)>+f_{j}(\hat{x})^{2}<\iota_{\hat{x}}^{(-1)}(u), \iota_{\hat{x}}^{(-1)}(v)>
$$

and

$$
\beta_{j}\left(u_{1}, \ldots, u_{n}\right):=f_{j}(\hat{x})^{m+n} \beta\left(\iota_{\hat{x}}\left(u_{1}\right), \ldots, \iota_{\hat{x}}\left(u_{n}\right)\right)
$$

depend only on $x$, and not on the choice of $\hat{x}$ in the fiber of $x$. Hence they give rise to a well-defined Riemannian metric $g_{j}$, as well as a volume form $\beta_{j}$ on $N_{j}$, which are easily checked to be invariant by local automorphisms. Because in the right-hand term of (4), all the maps involved are restrictions to $\hat{N}_{j}$ of continuous maps on $\hat{N}$, we get that $g_{j}$ admits a continuous extension to $\bar{N}_{i}$. To see that $\beta_{j}$ extends to a continuous $n$-form on $N$, we consider $\left(U_{i}, \alpha_{i}\right)_{i \in I}$ a smooth partition of unity of $N$. We assume the $U_{i}$ are chosen small enough so that there is a smooth section $\sigma_{i}: U_{i} \rightarrow \hat{N}$ for all $i \in I$. For every $x \in U_{i}$, and every $n$-uple $\left(u_{1}, \ldots, u_{n}\right)$ of $T_{x} N$, we define

$$
\tilde{\beta}_{j, U_{i}}\left(u_{1}, \ldots, u_{n}\right)=f_{j}(\hat{x})^{m+n} \beta\left(\iota_{\sigma_{i}(x)}\left(u_{1}\right), \ldots, \iota_{\sigma_{i}(x)}\left(u_{n}\right)\right)
$$

The form $\beta_{j, U_{i}}$ coincides with $\beta_{j}$ on $U_{i} \cap N_{j}$. Therefore, the $n$-form $\tilde{\beta}_{j}:=$ $\Sigma_{i \in I} \alpha_{i} \tilde{\beta}_{U_{i}}$ is continuous and restricts to $\beta_{j}$ on $N_{j}$. $\diamond$

We can now finish the proof of proposition 7.1. If $\left(M, \hat{M}, \omega^{M}\right)$ is not geometrically maximal, there exists a Cartan geometry $\left(N, \hat{N}, \omega^{N}\right)$ modelled on $\mathbf{X}$ such that $M \subsetneq N$ is a proper open subset, $\hat{M} \subset \hat{N}$ is a $P$-invariant open subset and $\omega^{M}$ is the restriction of $\omega^{N}$ to $\hat{M}$. Assume for a contradiction that $M$ is not flat. Then, keeping the notations of lemma 7.2, $\bigcup_{j=1}^{s} N_{j}$ is dense in $N$, so that $M \cap N_{j_{0}} \neq \emptyset$ for some integer $j_{0} \in\{1, \ldots, s\}$. Because $\operatorname{Aut}\left(M, \omega^{M}\right)$ acts transitively on $M$, the third point of lemma 7.2 ensures that $M \subset N_{j_{0}}$, and $\left(M, g_{j_{0}}\right)$ is a homogeneous Riemannian manifold, hence is complete. If $M \subsetneq N$, the topological boundary $\partial M$ of $M$ in $N$ is nonempty, and we can pick $x_{\infty} \in \partial M$ an accessible point. There exists a $C^{1}$-path $\alpha:\left[0,1\left[\rightarrow M\right.\right.$, such that $\lim _{t \rightarrow 1^{-}} \alpha(t)=x_{\infty}$. By completeness, the $g_{j_{0}}$-length of $\alpha$ is infinite. This contradicts the fact that $g_{j_{0}}$ extends continuously to $\bar{N}_{j_{0}} \cdot \diamond$

In the following statement, we endow $\operatorname{Aut}\left(M, \omega^{M}\right)$ with he topology of uniform convergence on compact subsets of $M$. For this topology, $\operatorname{Aut}\left(M, \omega^{M}\right)$ is a closed subgroup of $\operatorname{Homeo}(M)$ (observe that this statement is stronger than the one stated at the begining of section 6.1), and there is even a 
stronger property: if $\left(\varphi_{k}\right)$ is a sequence of $\operatorname{Aut}\left(M, \omega^{M}\right)$ which converges uniformly on some nonempty open subset $U \subset M$ to an injective continuous map $\varphi_{\infty}: U \rightarrow M$, then $\left(\varphi_{k}\right)$ converges in $\operatorname{Aut}\left(M, \omega^{M}\right)$. This fact is proved in [Fr1, Theorem 3]. The next theorem gives an obstruction for a regular, rank one parabolic geometry, to admit a geometric compactification.

Theorem 7.3. Let $\left(M, \hat{M}, \omega^{M}\right)$ be a noncompact, regular, rank one parabolic geometry. Assume that $\left(M, \hat{M}, \omega^{M}\right)$ is not flat. If Aut $\left(\hat{M}, \omega^{M}\right)$ is noncompact, then $\left(M, \hat{M}, \omega^{M}\right)$ does not admit any geometric compactification.

Proof: Let us assume that $\left(M, \hat{M}, \omega^{M}\right)$ admits a geometric compactification $\left(N, \hat{N}, \omega^{N}\right)$. As in the proof of 7.1 , we assume that $M \subsetneq N$ is a proper open subset, $\hat{M} \subset \hat{N}$ is a $P$-invariant open subset and $\omega^{M}$ is the restriction of $\omega^{N}$ to $\hat{M}$. If $\left(M, \hat{M}, \omega^{M}\right)$ is not flat, there exists $j_{0}$ such that $M_{0}:=M \cap N_{j_{0}}$ is a nonempty open subset of $N$. By lemma 7.2, $\operatorname{Aut}\left(M, \omega^{M}\right)$ leaves $M_{0}$ invariant. Notice that $M_{0}$ might not be connected. Nevertheless, any connected component of $M_{0}$ must be charged by the $n$ form $\beta_{j_{0}}$, while, by the third point of lemma 7.2 , the total mass of $M_{0}$ with respect to $\beta_{j_{0}}$ is finite. Hence any component has to be left invariant by a finite index subgroup of $\operatorname{Aut}\left(M, \omega^{M}\right)$. Therefore, we may assume that $M_{0}$ is connected, keeping the assumption that $\operatorname{Aut}\left(M, \omega^{M}\right)$ is noncompact and leaves $M_{0}$ invariant. Let us denote by $\bar{M}_{0}$ the closure of $M_{0}$ in $N$ and by $\partial M_{0}$ its topological boundary. If $\alpha:[0,1] \rightarrow \bar{M}_{0}$ is a $C^{1}$-curve, let $L(\alpha)$ be its length with respect to $g_{j_{0}}$. For $x \in M_{0}$, we define

$$
d(x):=\inf \left\{L(\alpha) \mid \alpha:[0,1] \rightarrow \bar{M}_{0}, \alpha(0)=x, \alpha(1) \in \partial M_{0}\right\}
$$

One checks that $d$ is a continuous function on $M_{0}$. Because $g_{j_{0}}$ extends continuously to $\bar{M}_{0}$, if $\left(x_{k}\right)$ is a sequence of $M_{0}$ which converges to $x_{\infty} \in$ $\partial M_{0}$, then $d\left(x_{k}\right)$ tends to 0 . Hence, for $\epsilon>0, K_{\epsilon}:=d^{-1}([\epsilon,+\infty[)$ is a compact subset of $M_{0}$, and if $\epsilon$ is chosen small enough, $K_{\epsilon}$ has nonempty interior. Now, because $\operatorname{Aut}\left(M, \omega^{M}\right)$ acts on $M_{0}$ preserving the Riemannian metric $g_{j_{0}}$ and the compact set $K_{\epsilon} \subset M_{0}$, the remark just before theorem 7.3 ensures that $\operatorname{Aut}\left(M, \omega^{M}\right)$ is compact. $\diamond$

Observe that theorem 7.3 rules out the existence of geometric compactifications, but it is not excluded that some manifolds satisfying the hypotheses of the theorem admit nontrivial geometric embeddings. 


\section{Maximal conformally homogeneous Riemannian structures: proof of theorem 1.1}

Thanks to proposition 7.1, theorem 1.1 reduces to the determination of those homogeneous conformally flat Riemannian manifolds which are not conformally maximal. We know the list of homogeneous conformally flat Riemannian structures, thanks to D.V. Alekseevskii and B. N. Kimel'fel'd. Since compact Riemannian structures are obviously conformally maximal, we restate the result they obtained for noncompact manifolds. The notations are those adopted in theorem 1.1.

Theorem 8.1. $[A-K]$ Let $(M, g)$ be a connected, noncompact conformally flat Riemannian manifold of dimension $n \geq 3$, with a transitive group of conformal transformations. Then $(M, g)$ is conformally equivalent to one of the following spaces:

1. The real hyperbolic space $\mathbf{H}^{n}$.

2. The quotient of the Euclidean space $\mathbf{R}^{n}$ by a discrete group $\Gamma$ of translations.

3. The quotient of $\mathbf{H}^{n-1} \times \mathbf{R}$ by $\{i d\} \times \Gamma$, where $\Gamma$ is a discrete group generated by a translation of $\mathbf{R}$.

4. The quotient of $\mathbf{H}^{n-m} \times \mathbf{S}^{m}$ by $\{i d\} \times \Gamma$, where $\Gamma$ is a discrete group of isometries of $\mathbf{S}^{m}, n-1 \geq m \geq 2$.

We are going to show that most spaces described in theorem 8.1 are actually conformally maximal. By corollary 1.5, this is for instance the case of all spaces appearing in point 2, except for the Euclidean space $\mathbf{R}^{n}$. We are now going to check which are the non conformally maximal examples in points 3 and 4 of the theorem.

Recall that we see $\mathbf{S}^{n}$ as the projectivization of the null cone of $Q(x):=$ $-2 x_{0} x_{n+1}+x_{1}^{2}+\ldots+x_{n}^{2}$. For $1 \leq m \leq n-1$, let $E$ be the subspace of $\mathbf{R}^{n+2}$ spanned by $e_{0}, e_{n+1}, e_{1}, \ldots, e_{n-m-1}$, and $F$ the subspace spanned by $e_{n-m}, \ldots, e_{n}$. If $H^{+}$is one connected component of $\{Q=-1\} \cap E$ and $S=F \cap\{Q=+1\}$, then $H^{+} \times S$, endowed with the metric induced by $Q$ is isometric to $\mathbf{H}^{n-m} \times \mathbf{S}^{m}$. We still adopt the convention that $\mathbf{H}^{1}$ is the 1-dimensional Euclidean space, and that $\mathbf{S}^{1}$ is the circle endowed with the homogeneous Riemannian metric of total length $2 \pi$. The map $j:(u, v) \rightarrow[u+v]$ is a conformal embedding from $H^{+} \times S$ into $\mathbf{S}^{n}$. Let 
us denote by $\Omega_{m}$ the image of $j$. The boundary of $\Omega_{m}$ in $\mathbf{S}^{n}$ is the projectivization of the null cone of $Q_{\mid E}$. It is a sphere $\Sigma_{m}$ of dimension $n-m-1$ (if $m=n-1$, it is the union of two points). In particular, $\Sigma_{m}$ is a smooth submanifold of codimension greater or equal to 2 , and the domain $\Omega_{m}$ is normal. The conformal group of $\Omega_{m}$ is the subgroup of $P O(1, n+1)$ preserving the splitting $E \oplus F$. It is a product $(O(1, n-m) \times O(m+1)) /\{ \pm I d\}$, and the factor $O(m+1)$ fixes $\Sigma_{m}$ pointwise. Theorem 1.4 directly implies:

Lemma 8.2. If $\Gamma$ is a nontrivial discrete subgroup of $O(m+1)$, and if a manifold $M$ is the quotient of $\Omega_{m}$ by the group $\{i d\} \times \Gamma \subset O(1, n-m) \times$ $O(m+1)$, then $M$ is conformally maximal.

Let us consider the product $\mathbf{H}^{n-1} \times \mathbf{R}$. By the previous discussion, it is the conformal universal cover of $\Omega_{1}$, and the fundamental group of $\Omega_{1}$ acts on $\mathbf{H}^{n-1} \times \mathbf{R}$ by the translation $(x, t) \mapsto(x, t+2 \pi)$. The sphere $\Sigma_{1}$ has codimension 2 , and if $x_{\infty} \in \Sigma_{1}$, the image of $\Sigma_{1} \backslash\left\{x_{\infty}\right\}$ by a stereographic projection of pole $x_{\infty}$ is an affine space of codimension 2 in $\mathbf{R}^{n}$. It is then easily checked that $\Omega_{1}$ satisfies the strong version of property $(H)$, as stated at the begining of corollary 6.6. If $\Gamma$ is the subgroup of $\operatorname{Is}(\mathbf{R})$ generated by a translation $t \mapsto t+\tau$, then corollary 6.6 tells us that the quotient of $\mathbf{H}^{n-1} \times \mathbf{R}$ by $\{i d\} \times \Gamma$ is conformally maximal, except maybe if $2 \pi=k \tau$ for some integer $k$. By lemma 8.2, for every integer $k \neq 1$, the quotient we get is conformally maximal. We conclude that in the case 3 of theorem 8.1, the only example which is not conformally maximal is obtained when the group $\Gamma$ is generated by $t \mapsto t+2 \pi$, and corresponds to the product $\mathbf{H}^{n-1} \times \mathbf{S}^{1}$. Finally, lemma 8.2 also implies that all examples of point 4 in theorem 8.1 are conformally maximal, except $\mathbf{H}^{n-m} \times \mathbf{S}^{m}$.

The above case by case study finish the proof of theorem 1.1.

\section{$9 \quad C R$-maximal homogeneous structures}

The aim of this last section is to prove the analogue of theorem 1.1 for $C R$-structures.

Theorem 9.1. Let $M^{2 n+1}, n \geq 1$, be a homogeneous, strictly pseudoconvex $C R$-manifold. If it is not $C R$-maximal, then it is $C R$-equivalent to a homogeneous open subset of the flat $C R$-sphere.

As for the conformal case, we infer from proposition 7.1 that a noncompact $C R$-structure which is homogeneous, and which is not $C R$-maximal must be $C R$-flat. Hence we must find out which homogeneous $C R$-flat manifolds are 
not maximal. Our study here will rely on the classification of homogeneous domains of the flat $C R$-sphere by D. Burns and S. Schnider in [BS].

Before stating the results of [BS], let us recall some basic facts about flat $C R$-geometry, and the projective model of the flat $C R$-sphere $\mathbf{S}^{2 n+1}$ (the reader will find more details in [Go, chap. 4] and [BS]). Let $\mathbf{C}^{1, n+1}$ denote the vector space $\mathbf{C}^{n+2}$ endowed with the lorentz hermitian form

$$
Q(z):=2 \Im m\left(z_{0} \bar{z}_{n+1}\right)+\left|z_{1}\right|^{2}+\ldots+\left|z_{n}\right|^{2}
$$

Let $\mathcal{C}$ be the null cone of $Q$, with the origin removed. Then, the projectivization of $\mathcal{C}$ in $\mathbf{C} \mathbf{P}^{n+1}$ is a smooth sphere $\mathbf{S}^{2 n+1}$, endowed with a natural $C R$-structure. If $U(1, n+1)$ denotes the subgroup of $G L(n+2, \mathbf{C})$ which preserves $Q$, then the automorphism group of this $C R$-structure is exactly $G:=P U(1, n+1)$ (the quotient of $U(1, n+1)$ by the linear homothetic transformations). We already mentioned in section 7 that the Lie algebra $\mathfrak{g}$ has the following structure: $\mathfrak{g}=\mathfrak{g}_{-2} \oplus \mathfrak{g}_{-1} \oplus \mathfrak{a} \oplus \mathfrak{k} \oplus \mathfrak{g}_{+1} \oplus \mathfrak{g}_{+2}$. Let $\nu$ be the point of $\mathbf{S}^{2 n+1}$ defined by $\left[e_{0}\right]$ (the projectivization of the vector $\left.e_{0}\right)$, and $o:=\left[e_{n+1}\right]$. The stabilizer $P$ of $\nu$ in $G$ is a semi-direct product $(A \times K) \ltimes N^{+}$, where $A, K$ and $N^{+}$have Lie algebras $\mathfrak{a}, \mathfrak{k}$ and $\mathfrak{g}_{+1} \oplus \mathfrak{g}_{+2}$ respectively. Moreover, $A$ is isomorphic to $\mathbf{R}_{+}^{*}, K$ is isomorphic to $U(n)$, and $\mathrm{N}^{+}$is isomorphic to the $2 n+1$-dimensional Heisenberg group. The product $A \times K$ is the subgroup of $G$ fixing both $o$ and $\nu$.

Let us cal $\mathcal{H}$ eis $(2 n+1):=\mathfrak{g}_{+1} \oplus \mathfrak{g}_{+2}$. The map $s: \mathcal{H}$ eis $(2 n+1) \rightarrow \mathbf{S}^{2 n+1} \backslash$ $\{\nu\}$, defined by $s(\xi):=\exp _{G}(\xi) . o$ is a diffeomorphism called stereographic projection. We thus see $\mathcal{H} e i s(2 n+1)$ as an open subset of $\mathbf{S}^{2 n+1}$, endowed with the induced flat $C R$-structure. The stereographic projection allows to read the natural action of $P$ on $\mathbf{S}^{2 n+1} \backslash\{\nu\}$ as an action of $P$ on $\mathcal{H} e i s(2 n+1)$ (which moreover, turns out to be affine). The group $N^{+}$acts freely and transitively on $\mathcal{H}$ eis $(2 n+1)$, and the action of $\mathbf{R}_{+}^{*} \times U(n)$ is linear: if we identify $\mathcal{H} e i s(2 n+1)$ with $\mathbf{C}^{n} \oplus \mathbf{R}$, and denote by $(u, t) \in \mathbf{C}^{n} \times \mathbf{R}$ its elements, then $\lambda .(u, t)=\left(\lambda u, \lambda^{2} t\right)$ for $\lambda \in \mathbf{R}_{+}^{*}$, and $L .(u, t)=(L . u, t)$ for $L \in U(n)$.

A $m$-dimensional complex linear subspace $V_{m} \subset \mathbf{C}^{1, n+1}$ is lorentz-hermitian if the restriction of the form $Q$ to $V$ has type $(1, m-1)$, for $2 \leq m \leq n+2$. We then denote by $\Sigma_{V_{m}}$ the projectivization in $\mathbf{C P}^{n+1}$ of the set $V_{m} \cap \mathcal{C}$. It is a smooth subsphere of $\mathbf{S}^{2 n+1}$, of dimension $2 m-3$.

By $V_{\mathbf{R}}$, we denote the span over $\mathbf{R}$ of the vectors $e_{0}, \ldots, e_{n+1}$. The set $\Sigma_{V_{\mathbf{R}}}$ is the projectivization of $V_{\mathbf{R}} \cap \mathcal{C}$. It is a smooth $n$-dimensional subsphere of $\mathbf{S}^{2 n+1}$.

For $1 \leq m \leq n-1$, we define $\mathfrak{l}_{m}:=\left\{(u, t)=\left(u_{1}, \ldots, u_{m}, u_{m+1}, \ldots, u_{n}, t\right) \in\right.$ $\mathcal{H}$ eis $\left.(2 n+1) \mid u_{1}, \ldots, u_{m} \in \mathbf{C}, u_{m+1}, \ldots, u_{n} \in \mathbf{R}, t \in \mathbf{R}\right\}$. If $m=0, \mathfrak{l}_{0}$ 
comprises all the $(u, t) \in \mathcal{H} \operatorname{eis}(2 n+1)$ such that $u \in \mathbf{R}^{n}$. The connected Lie subgroup of $G$ with Lie algebra $\mathfrak{l}_{m}$ will be denoted by $L_{m}$ in the sequel. We can now describe the classification obtained by Burns and Schnider.

Theorem 9.2. [BS, Theorem 3.1]

The noncompact homogeneous domains of the $C R$-sphere $\mathbf{S}^{2 n+1}$ are $C R$ diffeomorphic to one of the following spaces.

1. The domain $D_{m}:=\mathbf{S}^{2 n+1} \backslash \Sigma_{V_{m}}$, where $V_{m} \subset \mathbf{C}^{1, n+1}$ is a m-dimensional lorentz-hermitian subspace, $2 \leq m \leq n+1$.

2. The space $\mathbf{S}^{2 n+1} \backslash \Sigma_{V_{\mathbf{R}}}$.

3. The space $\mathcal{H} e i s(2 n+1)$.

4. A connected component $D_{m}^{\prime}$ of the space $\mathcal{H}$ eis $(2 n+1) \backslash L_{m} .0,0 \leq m \leq$ $n-1$.

Let $M$ be noncompact homogeneous $C R$-manifold of dimension $2 n+1$. We assume that $M$ is not $C R$-maximal. Then proposition 7.1 ensures that $M$ is $C R$-flat. We can then use [BS, proposition 1.4]: $M$ has the same $C R$ universal cover than a noncompact homogeneous domain of $\mathbf{S}^{2 n+1}$. We now begin a case by case study using theorem 9.2 above.

Assume we are in the first case of theorem 9.2. The set $\Sigma_{V_{m}}$ is diffeomorphic to a sphere of codimension $2(n+2-m)$ in $\mathbf{S}^{2 n+1}$. If $2 \leq m<n+1$, the domain $D_{m}$ is simply connected, hence $C R$-diffeomorphic to the universal cover $\tilde{M}$. The group of $C R$-automorphisms of $D_{m}$ is exactly the subgroup $H \subset P U(1, n+1)$ which preserves the splitting $\mathbf{C}^{1, n+1}=V_{m} \oplus V_{m}^{\perp}$ (where $V_{m}^{\perp}$ denotes the orthogonal of $V_{m}$ with respect to the hermitian form $Q$ ). Hence $H$ is the subgroup $P U(1, m-1) \times U(n+2-m)$ and $M$ is $C R$ diffeomorphic to a quotient of $D_{m}$ by a discrete subgroup $\Gamma \subset H$. We will use several times the following lemma.

Lemma 9.3. Let $\mathbf{K}$ be the field of real or complex numbers. Let $\Gamma$ be a nontrivial subgroup of isometries of $\mathbf{H}_{\mathbf{K}}^{m}$, the $m$-dimensional hyperbolic space over $\mathbf{K}$. We assume that $m \geq 2$ if $\mathbf{K}=\mathbf{R}$, and $m \geq 1$ if $\mathbf{K}=\mathbf{C}$. Then the centralizer $Z(\Gamma)$ of $\Gamma$ in $I s\left(\mathbf{H}_{\mathbf{K}}^{m}\right)$ does not act transitively on $\mathbf{H}_{\mathbf{K}}^{m}$.

Let us postpone the proof of the lemma for a moment. Because $M$ is homogeneous, lemma 9.3 ensures that the group $\Gamma \subset H$ must actually be a subgroup of $\{i d\} \times U(n+2-m)$. Now, it is easily checked that this group $\{i d\} \times U(n+2-m)$ fixes pointwise $\Sigma_{V_{m}}$, so that according to theorem 1.4, 
the only possibility for $M$ not being $C R$-maximal is the triviality of $\Gamma$. When $m=n+1, D_{m}$ is not simply connected. Nevertheless, the image of $\Sigma_{V_{m}} \backslash\{\nu\}$ by the stereographic projection $s^{-1}$ is a codimension 2 real affine subspace of $\mathcal{H}$ eis $(2 n+1)$. We infer that the fundamental group of $D_{m}$ is $\mathbf{Z}$, and moreover, the hypotheses of corollary 6.6 are satisfied. Corollary 6.6 ensures that $M$ must be $C R$-diffeomorphic to a quotient of $\mathbf{S}^{2 n+1} \backslash \Sigma_{V_{m}}$ and we conclude as above that the quotient is actually trivial.

We assume now that we are in the second case of theorem 9.2. The space $\mathbf{S}^{2 n+1} \backslash \Sigma_{V_{\mathbf{R}}}$ can be identified to the unit tangent bundle of the real hyperbolic space $\mathbf{H}_{\mathbf{R}}^{n+1}$, and the group of $C R$ automorphisms is $P O(1, n+1)$ ([BS, Proposition 6.1]). In particular, lemma 9.3 implies that no nontrivial quotient of $\mathbf{S}^{2 n+1} \backslash \Sigma_{V_{\mathbf{R}}}$ by a discrete subgroup $\Gamma \subset P O(1, n+1)$ is homogeneous. If $n \geq 2, \mathbf{S}^{2 n+1} \backslash \Sigma_{V_{\mathbf{R}}}$ is simply connected, and the remarks above imply that $M$ is $C R$-diffeomorphic to $\mathbf{S}^{2 n+1} \backslash \Sigma_{V_{\mathbf{R}}}$. If $n=1, \Sigma_{V_{\mathbf{R}}}$ is a circle in $\mathbf{S}^{3}$. Using again a stereographic projection, we check that we are under the hypotheses of corollary 6.6. By the first point of this corollary, $M$ must be a quotient of $\mathbf{S}^{3} \backslash \Sigma_{V_{\mathbf{R}}}$. As above, homogeneity of $M$ implies that this quotient is trivial.

The case where $M$ is covered by $\mathcal{H}$ eis $(2 n+1)$ was dealt with in section 5.2.5. The hypothesis that $M$ is not $C R$-maximal leads to the conclusion that $M$ must be $C R$-diffeomorphic to $\mathcal{H} e i s(2 n+1)$.

It remains to deal with the case where $M$ has the same $C R$-universal cover as $\mathcal{H} e i s(2 n+1) \backslash L_{m} .0$ (this later space is not connected when $m=n-1$, in which case we consider only one component). We will use the:

Lemma 9.4. Let $\Gamma \subset\left(\mathbf{R}_{+}^{*} \times U(m) \times O(n-m)\right) \ltimes L_{m}$ be a discrete subgroup. If the quotient of $\mathcal{H}$ eis $(2 n+1) \backslash L_{m} .0$ by $\Gamma$ is homogeneous, but not $C R$ maximal, then $\Gamma$ is trivial.

Proof: If the quotient of $\mathcal{H} e i s(2 n+1) \backslash L_{m} .0$ by $\Gamma$ is homogeneous, there must exist a connected subgroup $L \subset\left(\mathbf{R}_{+}^{*} \times U(m) \times O(n-m)\right) \ltimes L_{m}$ which centralizes $\Gamma$ and acts transitively on $D_{m}^{\prime}$. From [BS, lemma 3.3], we infer that $L$ then acts transitively on $\mathcal{H} e i s(2 n+1) \backslash L .0$. Hence $L .0=L_{m} .0$, which implies $L_{m} \subset L$. We get that the action of $L$ is transitive on $\partial D_{m}^{\prime}$, the boundary of $D_{m}^{\prime}$ in $\mathcal{H} e i s(2 n+1)$. Let us also observe that the component of $L$ on the $\mathbf{R}_{+}^{*}$-factor in $\left(\mathbf{R}_{+}^{*} \times U(m) \times O(n-m)\right) \ltimes L_{m}$ is nontrivial. Indeed, if it were not the case, $L$ would be a subgroup of $U(n) \ltimes H e i s(2 n+$ $1)$, and this latter group acts transitively on $\mathcal{H}$ eis $(2 n+1)$ with compact 
stabilizers, hence preserves a Riemannian metric on $\mathcal{H}$ eis $(2 n+1)$. Then, the function "distance to $\partial D_{m}^{\prime}$ " would be preserved by $L$, contradicting the homogeneity of $D_{m}^{\prime}$ under the action of $L$. Any element of $L$ having a nontrivial component on the $\mathbf{R}_{+}^{*}$-factor has a unique fixed point $q \in \partial D_{m}^{\prime}$. Because $L$ centralizes $\Gamma$, each element of $\Gamma$ fixes $q$, and because $L$ acts transitively on $\partial D_{m}^{\prime}, \Gamma$ actually fixes $\partial D_{m}^{\prime}$ pointwise. We infer from theorem 1.4 that any homogeneous quotient of $D_{m}^{\prime}$ is $C R$-maximal, except $D_{m}^{\prime}$ itself. $\diamond$

Lemma 9.4 ensures that whenever $D_{m}^{\prime}$ is simply connected, a homogeneous $C R$-manifold $M$ having the same universal cover as $D_{m}^{\prime}$ and which is not $C R$-maximal is $C R$-diffeomorphic to $D_{m}^{\prime}$. It remains to consider the case $m=n-2$, which is the only one for which $D_{m}^{\prime}$ is not simply connected. In this case $L_{n-2} .0 \cup\{\nu\}$ is a topological sphere of codimension 2 in $\mathbf{S}^{2 n+1}$. This sphere is smooth except at the point $\nu$ which is singular. Because $L_{n-2} .0$ is a codimension 2 linear subspace of $\mathcal{H}$ eis $(2 n+1)$, we see that the fundamental group of $D_{n-2}^{\prime}$ is $\mathbf{Z}$. We also check that the hypotheses of corollary 6.6 are satisfied. Hence, this corollary implies that if $M$ is not $C R$-maximal, it must be a quotient of $D_{n-2}^{\prime}$ by a discrete subgroup of $\left(\mathbf{R}_{+}^{*} \times U(n-2) \times O(2)\right) \ltimes L_{n-2}$, and lemma 9.4 above ensures that $M$ must be $C R$-diffeomorphic to $D_{n-2}^{\prime}$.

The case by case study made above yields theorem 9.1.

\section{Proof of lemma 9.3}

The proofs for $\mathbf{K}=\mathbf{R}$ and $\mathbf{K}=\mathbf{C}$ are similar, so that we will deal only with the case $\mathbf{K}=\mathbf{C}$. We will also assume $m \geq 2$ since the case $\Gamma \subset$ $S U(1,1)$ is easily settled: no hyperbolic surface, except the hyperbolic disc, is homogeneous. Observe also that $Z(\Gamma)$ acts transitively on $\mathbf{H}_{K}^{m}$ if and only if $Z(\Gamma)_{0}$ does, so that it is enough to show that the conclusion of the lemma holds for $Z(\Gamma)_{0}$.

Because $\mathbf{H}_{\mathbf{K}}^{m}$ is a CAT(0) space, we know (see for instance [BH, Part II, Corollary 2.8]) that whenever $\Gamma$ is relatively compact in $\operatorname{Is}\left(\mathbf{H}_{\mathbf{K}}^{m}\right)$, its set of fixed points is a nonempty proper subset $\operatorname{Fix}(\Gamma) \subsetneq \mathbf{H}_{\mathbf{K}}^{m}$ (recall that $\Gamma$ is assumed to be nontrivial). Because $Z(\Gamma)_{0}$ has to preserve $\operatorname{Fix}(\Gamma)$, it can not act transitively on $\mathbf{H}_{\mathrm{K}}^{m}$ in this case.

We assume now that $\Gamma$ is not relatively compact in $\operatorname{Is}\left(\mathbf{H}_{\mathbf{K}}^{m}\right)$. It thus contains a sequence $\left(\gamma_{k}\right)$ leaving every compact subset. Let us consider the action of $\left(\gamma_{k}\right)$ on the boundary at infinity $\mathbf{S}^{2 m-1}=\partial \mathbf{H}_{\mathbf{K}}^{m}$. Considering if necessary a subsequence, $\left(\gamma_{k}\right)$ has a "North-South dynamics". It means that there exist two points $p^{+}$and $p^{-}$in $\partial \mathbf{H}_{\mathbf{K}}^{m}$ such that every point of $\partial \mathbf{H}_{\mathbf{K}}^{m} \backslash\left\{p^{-}\right\}$is 
attracted by $p^{+}$under the action of $\gamma_{k}$, and every point of $\partial \mathbf{H}_{\mathbf{K}}^{m} \backslash\left\{p^{+}\right\}$is attracted by $p^{-}$under the action of $\gamma_{k}^{-1}$ (observe that the points $p^{+}$and $p^{-}$ may coincide). Hence, $Z(\Gamma)_{0}$, the identity component of $Z(\Gamma)$, has to fix $p^{+}$ (and also $p^{-}$if $\left.p^{-} \neq p^{+}\right)$. Up to conjugacy into $\operatorname{Is}\left(\mathbf{H}_{\mathbf{K}}^{m}\right)$, the group $Z(\Gamma)_{0}$ is a subgroup of the parabolic group $P=\left(\mathbf{R}_{+}^{*} \times U(m-1)\right) \ltimes H e i s(2 m-1)$. If the projection on the $\mathbf{R}_{+}^{*}$ factor is trivial, then the action of $Z(\Gamma)_{0}$ is not transitive on $\mathbf{H}_{\mathbf{K}}^{m}$ (the orbits are "trapped" between two Heis $(2 m-1)$ orbits, i.e two horospheres). If the projection on the $\mathbf{R}_{+}^{*}$ factor is nontrivial, then some 1-parameter subgroup $\left\{\phi^{t}\right\}$ of $Z(\Gamma)_{0}$ must be conjugated into the reductive part $\mathbf{R}_{+}^{*} \times U(m-1)$, hence fixes exactly two distinct points $o$ and $\nu$ on $\partial \mathbf{H}_{\mathbf{K}}^{m}$. An index two subgroup $\Gamma^{\prime} \subset \Gamma$ thus fixes $o$ and $\nu$, hence is a subgroup of $\mathbf{R}_{+}^{*} \times U(m-1)$. Because $\Gamma^{\prime}$ is not relatively compact, its projection on the $\mathbf{R}_{+}^{*}$-factor is nontrivial, and we get that some element $\gamma^{\prime} \in \Gamma^{\prime}$ fixes exactly the two points $o$ and $\nu$. The same will then be true for the whole group $Z(\Gamma)_{0}$. We infer that $Z(\Gamma)_{0}$ leaves invariant the geodesic of $\mathbf{H}_{\mathbf{K}}^{m}$ joining $o$ and $\nu$, and in particular $Z(\Gamma)_{0}$ can not act transitively on $\mathbf{H}_{\mathbf{K}}^{m}$.

\section{Acknowledgment}

I would like to thank the referees for their very helpful remarks.

This work was supported by the ANR Geodycos.

\section{References}

[A-K] D.V. Alekseevskii, B. N. Kimel'fel'd, Classification of homogeneous conformally flat riemannian manifolds. Mathematical notes 24, no. 1, 559-562.

[BH] M.Bridson, A. Haefliger, Metric spaces of non-positive curvature. Grundlehren der Mathematischen Wissenschaften, 319. SpringerVerlag, Berlin, 1999.

[Bi] O. Biquard, Métriques d' Einstein asymptotiquement symétriques (Asymptotically symmetric Einstein metrics). Astérisque 265. Paris, Société Mathématique de France, 109 pp., 2000.

[Bo] B. Bosshard, On b-Boundaries of Special Space-Time Models. General Relativity and Gravitation 12 (1979), no 12, 963-966.

[BS] D. Burns, S. Shnider, Spherical hypersurfaces in complex manifolds. Invent. Math. 33 (1976), no. 3, 223246. 
[CS] A. Cap, H. Schichl, Parabolic geometries and canonical Cartan connections. Hokkaido Math. J. 29 (2000), no. 3, 453-505.

[C-H] G. Carron, M. Herzlich, The Huber theorem for non-compact conformally flat manifolds. Comment. Math. Helv. 77 (2002), no. 1, 192-220.

[C-H2] G.Carron, M. Herzlich, Conformally flat manifolds with nonnegative Ricci curvature. Compos. Math. 142 (2006), no. 3, 798-810.

[Car] E. Cartan, Sur les variétés à connexion projective. Bull. Soc. Math. France 52 (1924), 205-241.

[Cha] S.Y.A Chang; J. Qing; P.C Yang, Compactification of a class of conformally flat 4-manifold. Invent. Math. 142 (2000), no. 1, 6593.

[Ch] S.S.Chern; J.Moser, Real hypersurfaces in complex manifolds. Acta Math. 133 (1974), 219-271.

[Cla] C. J. S. Clarke, Space-Time Singularities. Commun. math. Phys. 49 (1976), 17-23.

[Cl] Y.H. Clifton, On the completeness of Cartan connections. J. Math. Mech. 16 (1966), 569-576.

[D] T.A Drumm, Fundamental polyhedra for Margulis space-times. Topology 31 (1992), no. 4, 677-683.

[Fr1] C. Frances, Sur le groupe d'automorphismes des géométries paraboliques de rang un. Annales Scientifiques de l'École Normale Supérieure. 40 (2007), no 5, 741-764.

[Fr2] C. Frances, Lorentzian Kleinian groups. Comment. Math. Helv. 80 (2005), no 4, 883-910.

[Fr3] C. Frances, The conformal boundary of Margulis space-times. C. R. Math. Acad. Sci. Paris 336 (2003), no. 9, 751-756.

[GL] C. R. Graham, J. M. Lee, Einstein metrics with prescribed conformal infinity on the ball. Adv. Math. 87 (1991), 186-225.

[Go] W.M Goldman, Complex hyperbolic geometry. Oxford Mathematical Monographs. Oxford Science Publications. The Clarendon Press, Oxford University Press, New York, 1999. 316 pp. 
[H] V. Hlavatý, Zur Konformgeometrie. I: Eichinvariante Konnexion. Proc. Akad. Amsterdam 38, 281-286 (1935).

[He1] M. Herzlich, Compactification conforme des variétés asymptotiquement plates. [Conformal compactification of asymptotically flat manifolds] Bull. Soc. Math. France 125 (1997), no. 1, 55-91.

[He2] M. Herzlich, The canonical Cartan bundle and connection in CR geometry. Math. Proc. Cambridge Philos. Soc. 146 (2009), no. 2, $415-434$.

$[\mathrm{J}] \quad$ R.A Johnson, The bundle boundary for the Schwarzschild and Friedmann solutions . General Relativity and Gravitation 12 (1979), no 12, 967-968.

[Ka] M. Kapovich, Hyperbolic manifolds and discrete groups. Progress in Mathematics, 183. Birkhauser Boston, Inc., Boston, MA, 2001.

[Ki] Seongtag Kim, An obstruction to the conformal compactification of Riemannian manifolds. Proceedings of the AMS. 128 (1999), no $6,1833-1838$.

[Kn] A,W Knapp, Lie groups beyond an introduction. Second edition. Progress in Mathematics, 140. Birkhuser Boston, Inc., Boston, MA, 2002.

[Ko] S. Kobayashi, Transformation groups in differential geometry. Reprint of the 1972 edition. Classics in Mathematics. SpringerVerlag, Berlin, 1995.

[KP1] R.S Kulkarni, U.Pinkall, Uniformization of geometric structures with applications to conformal geometry. Differential geometry, Peñiscola 1985, 190-209, Lecture Notes in Math., 1209, Springer, Berlin, 1986.

[KP2] R.S Kulkarni, U.Pinkall, A canonical metric for Möbius structures and its applications. Math. Z. 216 (1994), no. 1, 89-129.

[Ma] G.A Margulis, Free completely discontinuous groups of affine transformations. Dokl. Akad. Nauk SSSR 272 (1983), no. 4, 785788 .

[MK1] B. McKay, Complete complex parabolic geometries. International Mathematics Research Notices (2006). 
[MK2] B. McKay, Complete projective connections. arXiv:math/0504082v5.

[Mo] P. Montel, Leçons sur les familles normales de fonctions analytiques et leurs applications. 1974, Chelsea Pub. Co.

[Ob] M. Obata, The conjectures on conformal transformations of Riemannian manifolds. J. Differential Geometry 6 (1971/72), 247258.

[S] B.G Schmidt, A new definition of conformal and projective infinity of space-times. Comm. Math. Phys. 36 (1974), 73-90.

[S-Y] R.Schoen, S.T Yau, Conformally flat manifolds, Kleinian groups and scalar curvature. Invent. Math. 92 (1988), no. 1, 47-71.

[Sh] R.W. Sharpe, Differential Geometry: Cartan's generalization of Klein's Erlangen Program. New York: Springer, 1997.

[T] N.Tanaka, On non-degenerate real hypersurfaces, graded Lie algebras, and Cartan connections. Japan J. Math. 2 (1976). 131-190.

[Th] W.P Thurston, Three-dimensional geometry and topology. Vol. 1. Edited by Silvio Levy. Princeton Mathematical Series, 35. Princeton University Press, Princeton, NJ, 1997.

[Web] S.M Webster, On the transformation group of a real hypersurface. Trans. Amer. Math. Soc. 231 (1977), no. 1, 179-190.

Charles FRANCES

Laboratoire de Mathématiques, Bat. 425.

Université Paris-Sud.

91405 ORSAY.

Charles.Frances@math.u-psud.fr 